NASA Technical Memorandum 103703

AIAA-91-0130

\title{
The Aerodynamic Characteristics of Vortex Ingestion for the F/A-18 Inlet Duct
}

Bernhard H. Anderson

Lewis Research Center

Cleveland, Ohio

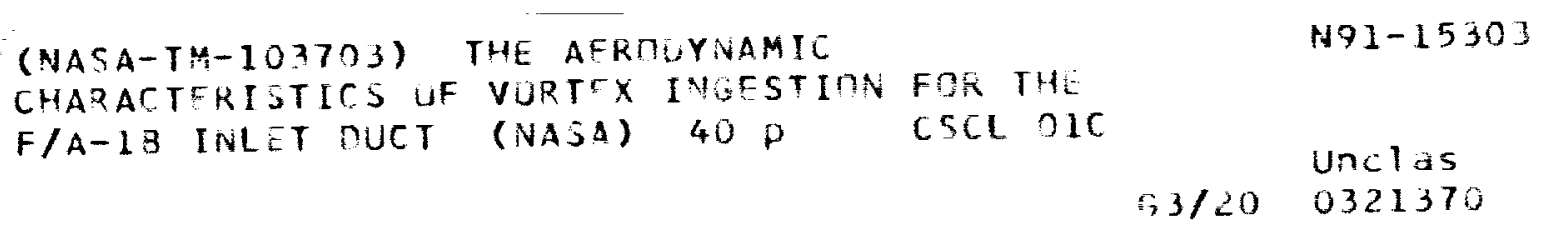

Prepared for the

29th Aerospace Sciences Meeting

sponsored by the American Institute of Aeronautics and Astronautics

Reno, Nevada, January 7-10, 1991

\section{N/Sก}




\title{
THE AERODYNAMIC CHARACTERISTICS OF VORTEX INGESTION FOR THE F/A-18 INLET DUCT
}

\author{
by \\ Bernhard H. Anderson \\ NASA Lewis Research Center \\ Cleveland, Ohio 44135
}

\begin{abstract}
A Reduced Navier Stokes (RNS) solution technique has been successfully combined with the concept of partitioned geometry and mesh generation to form a very efficient 3D RNS code aimed at the analysis-design engineering environment. Partitioned geometry and mesh generation is a pre-processor to augment existing geometry and grid generation programs which allows the solver to (1) recluster and existing gridfile mesh lattice, and (2) perturb an existing gridfile definition to alter the cross-sectional shape and inlet duct centerline distribution without returning to the external geometry and grid generator. The present results provide a quantitative validation of the initial value space marching 3D RNS procedure and demonstrates accurate predictions of the engine face flow field, with a separation present in the inlet duct as well as when vortex generators are installed to supress flow separation. The present results also demonstrate the ability of the 3D RNS procedure to analyze the flow physics associated with vortex ingestion in general geometry ducts such as the F/A-18 inlet. At the conditions investigated, these interactions are basically "inviscid like", i.e. the dominant aerodynamic characteristics have there origin in inviscid flow theory.
\end{abstract}

\section{INTRODUCTION}

Modern tactical aircraft are required to be maneuverable at subsonic, transonic, and supersonic speeds, without giving up good cruise performance. Consequently, proper integration of the engine inlet with the airframe is of paramount importance. Regarding the enhancement of inlet performance and operation, design for optimum airframe-inlet integration has the following goals: (a) to minimize approach flow angularity with respect to inlet cowl lip, (b) to deliver uniform, high pressure recovery flow to the inlet face, (c) to prevent or minimize vortex, wake, and boundary layer ingestion by the inlet over the flight envelope, (d) to reduce FOD/hot gas ingestion by the inlet, and finally (e) to minimize the potential for flow field interference from weapon carriage/firing, landing gear deployment, tanks, pods, or other hardware. The combination of inlet design and airframe integration must not only provide high pressure recovery to maintain the desired thrust levels, but also generate low flow distortion consistent with stable engine operation.

Engine face flow distortion is one of the most troublesome and least understood problems for designers of modern inlet engine systems, (Ref. 1 and 2). One issue is that there are numerous sources of flow field distortion that are ingested by the inlet or generated within the inlet duct itself. Among these sources are (a) flow separation at the cowl lip during maneuvering flight, (b) flow separation on the compression surfaces due to shock-wave boundary layer interactions, (c) spillage of the fuselage boundary layer into the inlet duct, (d) ingestion of aircraft vortices and wakes emanating from upstream disturbances, and (e) secondary flow and possibly flow separation within the inlet duct itself. Most developing aircraft have experienced one or more of these types of problems, particularly at high Mach numbers and/or extreme maneuver conditions, such that flow distortion at the engine face exceeded allowable limits. Such compatibility problems were encountered in the early versions of the B70, the F-111, the F-14, the MIG-25, the Tornado and the Airbus A300 to name a few examples. 
The effect of inlet distortion, whether it be pressure or temperature, steady or transient, is that the power available is reduced along with the engine-compression-system surge margin (i.e. the difference between the operating line and the surge line). Aeromechanical effects such as rotor-blade forced response and distortion effects on flutter boundaries have received less attention, so that a consensus on importance and state-of-the-art methodology have yet to emerge. Stability characteristics of current high performance turbofan engines are adversely affected by both spatial as well as temporal distortion. According to distortion theory, there are two kinds of temporal distortion: (1) transients characterized by discrete frequencies (deterministic), such as inlet buzz during supersonic flight, vortex or wake ingestion, and internal flow separation, and (2) random fluctions (stochastic), such as inlet-induced turbulence.

The availability of present generation of super computers has opened the possibility of using CFD to analyze, understand, and control some of the inlet-engine flow phenomena described. To this end, both Full Navier Stokes and Reduced Navier Stokes analyses are being developed. The overall approach is to develop fast and efficient FNS and RNS computational techniques, and use both approaches effectively to provide overall design-guidence information for future aircraft concepts. The FNS code under development for design-analysis uses the highly efficient multi-grid approach of Vatsa and Wedan, Ref. 3, and the design-guidence RNS code utilizes the general velocity-decomposition approach of Briley and McDonald, Refs. 4 and 5. The general approach to using Computational Fluid Dynamics to study inlet-engine compatibility issues are shown schematically in Figure 1, and is based on the of using both FNS and RNS analyses where appropriate. The Full Navier Stokes code is aimed at analysis of the inlet-forebody and internal duct flow field addressing such issues as (1) inlet inflow definition over a range of operating conditions and angles of attack, (2) detail description of the flow field in the vicinity of the cowl lip, (3) identification of sources of inlet flow unsteadyness such as shock boundary layer interactions, and internal flow separation, (4) analysis of internal flow structure within the transonic flow regime, (5) specialized interactions such as possible vortex breakdown within the inlet duct and, (6) unsteady internal interaction of a deterministic nature. The Reduced Navier Stokes anaysis addresses the inlet design issues such as (1) optimum duct geometry for a given inflow condition, (2) boundary layer control studies using devices such as vortex generators, (3) cross-sectional inlet duct shaping and its effect on total pressure recovery, engine face distortion, and transmission of disturbances through the inlet duct, and (4) distorton management control methods, particularily in high AOA aircraft configurations.

This paper represents one in a series of studies on the design issues associated with inletengine compatibility, which centers on the development of CFD tools and techniques which look promising within a analysis-design environment, and the application of these new analysis approaches to understand and control some of the phenomena encountered in inlet-engine compatibility. Specifically, this paper deals with three topics: (1) development and validation of subsonic RNS computational techniques for analysis of general geometry inlet ducts such as the F/A-18 configuration, (2) improvement of geometry and grid generation techniques for the analysis-design enviroment, (3) the aerodynamics of distortion and how the transmission of distortion through the F/A-18 inlet is effected by the geometric properties of the duct, where the inlet distortion arises from a vortex ingestion. 


\section{THEORETICAL BACKGROUND}

The reduced form of the Navier-Stokes equations originally termed parabolized NavierStokes equations (PNS) and more recently semi-elliptic, or partially parabolic are considered here as an initial-value space marching method for the evaluation of subsonic compressible flow with strong interactions and; or separation that arise from internal vortex flows. Techniques that use space marching with an approximate form of the Reduced Navier-Stokes equations (RNS), namely initial-value methods and those that require three-dimensional global iterations, have been considered for a number of years to predict flows in curved ducts and turbomachinery blade casades. Unfortunately, this terminology does not identify the revelant or significant physical approximations made and does not distinguish these approximations from the properties of the solution algorithm and the differential or difference equations. In other words, different methods within the same "category" will in some instances give significantly different results than what is reported herein.

Here, RNS methods are identified as either initial-value methods or methods which require global iterations. Although some type of forward-marching alogrithm may be used in both initial-value and global-iterations approaches, the present distinction is intended to emphasize that initial-value methods provide a solution after a single forward-marching integration, while global iteration schemes provide a solution to the system of governing equations only upon convergence of the iteration process. This distinction between the two categories is important, since conclusions reached in this paper are based on satisfying the system of governing equations after a single forward marching integration. A single forward marching integration is the manner in which computations will be accomplished in an analysis-design engineering environment.

\section{General Information}

Three dimensional viscous subsonic flows in complex inlet duct geometries are investigated by a numerical procedure which allows solution by spatial forward marching integration, utilizing flow approximations from the velocity-decomposition approach of Briley and McDonald, (Refs. 4 and 5). The goal of this approach is to achieve a level of approximations that will yield accurate flow predictions, while reducing the labor of solution below that of the full Navier Stokes equations. The governing equations for this approach have been given previously for orthogonal coordinates, and the approach has been applied successfully to problems whose geometries can be fitted conveniently with orthogonal coordinates systems, (Ref. 6). However, geometries encountered in typical subsonic inlet ducts cannot be treated easily using orthogonal coordinates, and this lead to an extension of this approach by Levy, Briley, and McDonald, Ref. 7, to treat ducted geometries with nonorthogonal coordinates. The nonorthogonal capability has been validated over a wide range of inlet flow conditions by Towne, (Refs. 8 and 9), and Anderson, (Ref. 10).

\section{Partitioned Geometry and Grid Generation}

Although the analysis itself was general, the class of duct geometrics that could be analyzed was represented by superelliptic cross-sections normal to a reference line space curve having continous second derivatives. The description of the superelliptic cross-sections was specified by polynomials defined in terms of a marching parameter, $\tau$. In the present development, the geometry formulation of Ref. 7 is extended to cover general ducted geometries define by a externally generated gridfile. The geometry description within the gridfile is a "ducted" geometry which has a variable cross-sectional area and shape, and a centerline which is curved and possibly twisted. In addition, the duct described by the gridfile is considered to have a defined centerline with continuous second derivatives. The surface geometry is described in terms of cross-sectional planes which lie perpendicular to the duct centerline, and thus represent the flow area at each streamwise station. Since the inlet duct geometry definition has been reduced to a cross section specification which is placed perpendicular to a centerline space curve, then a number of grid and 
geometry pre-processing functions can be performed prior to applying the RNS flow solver. These pre-processing functions are shown schematically in Figure (2) and include: (1) reclustering the existing gridfile mesh points for more accurate solutions in regions of high shear, (2) redefinition of the centerline space curve to satisfy design constraints, and (3) perturbations of the cross-sectional shape to reflect specified design iterations. The approach taken here is to develop a geometry pre-processor to augment the existing geometry and grid generation programs for internal inlet duct configurations.

In solving the equations of fluid dynamics by numerical means, two major problem areas are encountered: the generation of the three dimensional geometry and grid, and the algorithm by which the governing equations are processed to obtain a solution. Grid generation envolves three processes: (1) description of the boundary surface, (2) construction of the grid lattice within the boundary surface, and (3) solution dependent adaptation or clustering of the grid lattice to accurately resolve regions of rapid solution variations, i.e. large shear.

Within this framework, the modern computational codes have separated the functions of grid generation and flow solution, i.e. the geometry and mesh construction has been divorced from the solver. This separating of the functions is a sensible choice within a fundamental research environment where the processes of grid generation and flow solution are destinctly different and where the design is fixed, i.e., where variations of the same geometric configuration need not be considered. However, in a analysis-design engineering environment, or even in analysis of many three dimensional viscous flows, large advantages can be gained by having the ability to modify an existing gridfile without returning to a separate geometry and mesh generator. For example, adaptive mesh clustering is a very powerful technique to effectively use a limited number of grid points to define the multiple length scales that can arise in fluid dynamics problems, such as boundary layer and inviscid core regions. In numerous problems, particularly in three dimensional space, the location of the regions requiring definition are not known a-priori, but emerge as the solution develops. A vortex ingestion-boundary layer interaction is a case in point. For such problems, adaptive mesh re-clustering of an existing gridfile, without the neccssity of returning to a separate mesh generator, becomes a very powerful computational technique.

Redefinition of the centerline space curve and perturbation of the cross-sectional shape of the existing gridfile inlet duct description can have important significance within a analysis-design environment, where analysis of many variations of the same basic geometry with different inflow conditions is often the design task. Special constraints can easily be imposed on the inlet duct geometry within the system of patitioned geometry and mesh generation. For example, the centerline definition can easily be altered without affecting the inlet flow area distribution. This is shown in Figure (2), where an inlet duct equivalent to the original F/A-18 configuration was constructed with a straight rather than an out-of-plane offset centerline. Likewise, the crosssectional shape can easily be modified, without affecting either the centerline space curve or flow area distibution. This also is shown in figure (2), where an equivalent circular cross-section F/A-18 inlet duct is presented. These are very powerful computational capabilities within an design environment.

\section{Reference Coordinate System}

In the present development, the primary flow direction and body fitted coordinated system are related to a smooth reference line associated with the geometry of interest. This reference line, usually chosen to be the duct centerline, is a space curve required to have continuous second derivatives. The planar surfaces normal to the reference line at each axial location intersect the duct surface and are identified as the transverse (cross-sectional) planes of the duct geometry. The primary flow direction is taken as perpendicular to the transverse plane at each axial location, and thus parallel to the reference line. The reference line thus provides a necessary link between the duct geometry and the flow approximations made to permit solution as an inial value problem. An orthogonal reference coordinate system is derived to fit the reference line. In this 
system, the transverse coordinate are "Cartesian" and lie in the transverse plane, and the axial coordinate is normal to the transverse plane. Although the orthogonal coordinate system does not fit the duct geometry, it is aligned with the primary flow direction and thus provides a convenient method for introducing the flow approximations in a simple form. The approximate equations are written in this orothogonal reference coordinate system. A nonorthogonal body fitted coordinate is then devised by transforming the transverse Cartesian coordinates to obtain new transverse coordinates which fit the intersection of the duct with the transverse normal planes, and by simultaneously transforming the axial coordinate to fit the duct geometry. Transformations from the body-fitted coordinate, $y_{j}$ to the computational orthogonal coordinates, $x_{i}$, built on the reference line are calculated as part of the body-fitted system. The governing equations are then solved following a Jacobian transformation to the body fitted coordinate system, i.e. $\frac{\partial y_{j}}{\partial x_{i}}$ Thus, for example:

$$
\frac{\partial u_{p}}{\partial x_{i}}=\frac{\partial y_{j}}{\partial x_{i}} \frac{\partial u_{p}}{\partial y_{j}}
$$

and in general

$$
\frac{\partial}{\partial x_{i}}=\frac{\partial y_{j}}{\partial x_{i}} \frac{\partial}{\partial y_{j}}
$$

\section{The Governing Equations}

The reduced form of the Navier Stokes equations are derived through approximations made relative to the orothogonal coordinate system built upon the reference line described in the previous section. The reference line specification links the flow approximations with the duct geometry. Transverse coordinate surfaces are planes normal to the reference line. Equations governing the primary flow velocity, $\bar{U}_{p}$, and a secondary vorticity, $\omega$ normal to the transverse coordinate surfaces are derived utilizing approximations which permit solution of the equations as an initial value problem. Terms representing diffusion normal to the transverse coordinate planes are neglected. Approximate pressure gradients are imposed in the streamwise momentum equation from an apriori known pressure field obtained from a solution of the potential flow in the duct. Secondary flow velocities are determined from scalar and vector potential calculations in transverse coordinate surfaces, once the primary velocity is known.

The analysis is based on the decomposition of the overall velocity vector field $\bar{U}$ into a primary flow velocity $\bar{U}_{p}$ and a secondary flow velocity $\bar{U}_{s}$. The overall or composite velocity is determined from the superposition

$$
\bar{U}=\bar{U}_{p}+\bar{U}_{s}
$$

The primary flow velocity is represented as

$$
\bar{U}_{p}=u_{p} \hat{i}_{n}
$$

where $\hat{i}_{n}$ is the normal to the transverse plane. The primary flow velocity $u_{p}$ is determined from the solution of the primary momentum equation. In general orthogonal coordinates, the primary momentum equation becomes 


$$
\begin{aligned}
\frac{\rho u_{p}}{h_{1}} \frac{\partial u_{p}}{\partial x_{1}} & +\frac{\rho v_{s}}{h_{2}} \frac{\partial u_{p}}{\partial x_{2}}+\frac{\rho w_{s}}{h_{3}} \frac{\partial u_{p}}{\partial x_{3}}+u_{p}\left(v_{s} K_{12}+w_{s} K_{13}\right)-v_{s}^{2} K_{21}-w_{s}^{2} K_{31}=-\frac{1}{h_{1}} \frac{\partial}{\partial x_{1}}\left(P_{1}+P_{v}\right) \\
& +(J R e)^{-1}\left[\frac{\partial}{\partial x_{2}} \frac{h_{1}^{2} h_{3}}{h_{2}} \mu \frac{\partial}{\partial x_{2}}\left(\frac{u_{p}}{h_{1}}\right)+h_{1}^{2} h_{3} \mu K_{12} \frac{\partial}{\partial x_{2}}\left(\frac{u_{p}}{h_{1}}\right)\right] \\
& +(J R e)^{-1}\left[\frac{\partial}{\partial x_{3}} \frac{h_{1}^{2} h_{2}}{h_{3}} \mu \frac{\partial}{\partial x_{3}}\left(\frac{u_{p}}{h_{1}}\right)+h_{1}^{2} h_{2} \mu K_{13} \frac{\partial}{\partial x_{3}}\left(\frac{u_{p}}{h_{1}}\right)\right]
\end{aligned}
$$

where $J=h_{1} h_{2} h_{3}$ and $K_{i j} \equiv\left(h_{i} h_{j}\right)^{-1} \frac{\partial h_{i}}{\partial x_{j}}$.

The streamwise pressure gradients are approximated from an inviscid flow obtained by an a priori analysis, and are corrected for viscous effects by a mean pressure gradient term which depends only on the streamwise coordinate. In equation (2.3), $P_{I}$ is an a priori known pressure field, and $P_{V}$ is the one-dimensional correction to account for the mean viscous pressure loss. For internal flows, $P_{V}$ is determined to ensure that the integral mass flux condition is satisfied:

$$
\dot{m}=\int_{A} \rho u_{p} d A=\operatorname{CONSTANT}
$$

The secondary velocity $\bar{U}_{s}$ is derived from scalar and vector surface potentials denoted as $\phi$ and $\psi$, respectively. For $\rho$ the density and $\rho_{0}$ a constant reference density, $\bar{U}_{s}$ is written as

$$
\bar{U}_{s}=\nabla_{s} \phi+\frac{\rho_{0}}{\rho} \nabla \times \hat{i}_{n} \psi
$$

where $\nabla_{s}$ is the surface gradient operator defined by

$$
\nabla_{s}=\nabla-\hat{i}_{n}\left(\hat{i}_{n} \cdot \nabla\right)
$$

The scalar and vector potential equations become

$$
\begin{gathered}
\frac{\partial}{\partial x_{2}} \rho \frac{h_{1} h_{3}}{h_{2}} \frac{\partial \phi}{\partial x_{2}}+\frac{\partial}{\partial x_{3}} \rho \frac{h_{1} h_{2}}{h_{3}} \frac{\partial \phi}{\partial x_{3}}=-\frac{\partial}{\partial x_{1}}\left(h_{2} h_{3} \rho u_{p}\right) \\
\frac{\partial}{\partial x_{2}}\left(\frac{h_{3}}{h_{1} h_{2}} \frac{1}{\rho} \frac{\partial h_{1} \psi}{\partial x_{2}}\right)+\frac{\partial}{\partial x_{3}}\left(\frac{h_{2}}{h_{1} h_{3}} \frac{1}{\rho} \frac{\partial h_{1} \psi}{\partial x_{3}}\right)=-h_{2} h_{3} \Omega
\end{gathered}
$$

and the vorticity transport equation in general orthogonal coordinates can be written

$$
\begin{aligned}
& \frac{\rho u_{p}}{\tilde{h}_{1}} \frac{\partial}{\partial x_{1}} h_{2} h_{3} \Omega+\frac{\partial}{\partial x_{2}} h_{3} \rho v_{s} \Omega+\frac{\partial}{\partial x_{3}} h_{2} \rho w_{s} \Omega \\
& \quad+\frac{\partial h_{3} w_{s}}{\partial x_{1}} \frac{\partial}{\partial x_{2}}\left(\frac{\rho u_{p}}{h_{1}}\right)-\frac{\partial h_{2} v_{s}}{\partial x_{1}} \frac{\partial}{\partial x_{3}}\left(\frac{\rho u_{p}}{h_{1}}\right)-\frac{\partial}{\partial x_{2}}\left(h_{3} \rho u_{p}^{2} K_{13}\right)+\frac{\partial}{\partial x_{3}}\left(h_{2} \rho u_{p}^{2} K_{12}\right) \\
& \quad+\frac{\partial \rho v_{s}}{\partial x_{2}} \frac{\partial v_{s}}{\partial x_{3}}-\frac{\partial \rho v_{s}}{\partial x_{3}} \frac{\partial v_{s}}{\partial x_{2}}+\frac{\partial \rho w_{s}}{\partial x_{2}} \frac{\partial w_{s}}{\partial x_{3}}-\frac{\partial \rho w_{s}}{\partial x_{3}} \frac{\partial w_{s}}{\partial x_{2}} \\
& \quad=R^{-1}\left[\frac{\partial}{\partial x_{2}} \frac{h_{3}}{h_{1} h_{2}} \frac{\partial}{\partial x_{2}}+\frac{\partial}{\partial x_{3}} \frac{h_{2}}{h_{1} h_{3}} \frac{\partial}{\partial x_{3}}\right]\left(\mu h_{1} \Omega\right)
\end{aligned}
$$

where the decomposed transverse velocity components are given in orthogonal coordinates by 


$$
\begin{gathered}
v_{s}=v_{\phi}+v_{\psi}=\frac{1}{h_{1}} \frac{\partial \phi}{\partial x_{2}}+\frac{1}{h_{1} h_{3}} \frac{1}{\rho} \frac{\partial h_{1} \psi}{\partial x_{3}} \\
w_{s}=w_{\phi}+w_{\psi}=\frac{1}{h_{3}} \frac{\partial \phi}{\partial x_{3}}-\frac{1}{h_{1} h_{2}} \frac{1}{\rho} \frac{\partial h_{1} \psi}{\partial x_{2}}
\end{gathered}
$$

The equation of state for a perfect gas is given by

$$
p=\rho R T
$$

where $\mathrm{R}$ is the gas constant and $\mathrm{T}$ is temperature. In the present study, the stagnation energy was assumed to be a known constant, $E_{o}$, and the energy equation can be omitted from consideration. For constant stagnation energy, the gas law can be written as

$$
P_{I}+P_{V}=\frac{\gamma-1}{\gamma} \rho\left(E_{o}-\frac{\bar{U} \cdot \bar{U}}{2}\right)
$$

where $\gamma$ is the specific heat ratio.

\section{The Turbulence Model}

The mixing length model used in this analysis employes an an eddy-viscosity formulation for the Reynolds stresses expressed in the form

$$
\rho v^{i^{\prime} v^{\prime \prime}}=-\frac{\mu_{T}}{R e} \frac{\partial v^{J}}{\partial x_{i}}
$$

where the effective viscosity $\mu_{T}$ is added to the laminar viscosity $\mu$. The turbulent viscosity is related to the mean flow variables by means of a mixing length distribution

$$
\frac{\mu_{T}}{\operatorname{Re}}=\rho \ell^{2}(2 \overline{\bar{e}}: \overline{\bar{e}})^{1 / 2}
$$

where $\overline{\bar{e}}$ is the mean flow rate of strain tensor defined by

$$
\overline{\bar{e}}=\frac{1}{2}\left[(\nabla \vec{v})+(\nabla \vec{v})^{T}\right]
$$

The mixing length $\ell$ is determined from the empherical relationship of McDonald and Camarata, Ref. 11, for equilibrium turbulent boundary layers, which can be written as

$$
\ell(\tilde{y})=0.09 \delta_{b} \tan h\left[\frac{\kappa \tilde{y}}{0.09 \delta_{b}}\right] D
$$

where $\delta_{b}$ is the local boundary layer thickness, $\kappa$ is the von Karman constant, and $\tilde{y}$ is the distance from the wall, and $\mathrm{D}$ is the sublayer damping factor defined by

$$
D=\sqrt{p} \frac{\left(y^{+}-\bar{y}^{+}\right)}{\sigma_{1}}
$$

where

$$
y^{+}=\frac{\tilde{y}\left(\frac{\tau}{\rho}\right)^{\frac{1}{2}}}{\left(\frac{\mu}{\rho}\right)}
$$


and $\mathrm{p}$ is the normal probability, $\tau$ is the local shear stress, $\bar{y}^{+}=23, \sigma_{1}=8.0$, and $\tilde{y}$ is the distance to the wall.

\section{The FLARE Approximation}

The analysis as presented here is applicable only when the primary velocity is not negative. Since "small" regions of reverse flow can arise in curved inlet ducts, the numerical method is locally modified to permit forward marching when the flow contains small regions of reverse flow. The technique used follows Reyhner and Flugge-Lotz, (Ref. 12), by adding small artificial convection at grid points where the primary flow is reversed. This is known as the FLARE approximation, after the authors of Reference 12. For thin regions of reverse flow, although the area of flow separation can be very large compared to the passage itself, the technique permits the analysis to proceed downstream beyond reattachment, confining the FLARE approximation to the separated region.

\section{Vortex Generator Model}

One of the most commonly used methods to control local boundary layer separation entails the placement of vortex generators upstream of the problem area. Vortex generators in use today are small wing sections mounted on the inside surface of the inlet duct or wing surface, inclined at an angle to the oncoming flow to generate a shed vortex. The generators are usually sized to local boundary layer height to allow for the best interaction between the shed vortex and boundary layer itself, and are usually placed in groups of two or more upstream of the problem area. The principle of boundary layer control by vortex generators relies on induced mixing between the external or core stream and the boundary layer region. This mixing is promoted by vorticies trailing longitudinally over the duct surface adjacent to the edge of the boundary layer. Fluid particles with high momentum in the stream direction are swepted along helical paths towards the duct surface to mix with and, to some extent, replace the low momentum boundary layer flow. This is a continuous process that provides a source of re-energization to counter the natural boundary layer growth caused by friction, adverse pressure gradients, and low energy secondary flow accumulation. There are two basic configurations of vortex generators. In one configuration, all the vortex generators are inclined at the same angle with respect to the oncoming flow direction. These are called co-rotating configurations because the shed vorticies rotate in the same direction. In the other configuration, the vortex generators are grouped in pairs inclined in the opposite direction to the flow, such that pairs of counter rotating shed vorticies are generated.

Co-rotating vortex generators are very competitive in reducing flow separation if the generators are properily selected and located. The main advantage of co-rotating type vortex generators are their downstream effectiveness resulting in more effective usage of the vortex energy within the affected boundary layer. According to design "wisdom", this type of vortex has a few special advantages when used within $S$-duct inlet configurations, namely: (1) the induced vortices will remain close to the wall; consequently a "cleaner" core flow will result, and (2) the induced vorticies will counteract the natural and often strong secondary flows which develop.

Counter-rotating, equal strength vortex generators have been used in a number of aircraft inlet ducts, the F/A-18 and the center inlet duct on the production 727 aircrafts, to name a few examples. This type of vortex generator is very effective in reducing flow separation if the vortex generators are placed slightly upstream of the region of separation. According to design "wis$\mathrm{dom}^{\prime \prime}$, the disadvantages of this type of generators, as compared to co-rotating generators, are that the induced vorticies tend to lift off the duct surface, thus reducing their effectiveness, causing higher loss in inlet recovery and larger total pressure distortion at the engine face.

The performance of vane-type vortex generators was evaluated by Taylor, (Ref. 13), for diffusers and airfoils at low speed, and by Valentine and Carrol, (Refs. 14 and 15), for airfoils and 
wings at high speeds. This work provides trends in effectiveness for certain vortex generator design variables such as angle-of-attack, height, distance ahead of separation, etc. Attention was, however, focused on the detailed changes that were produced in the boundary layer as a result of placement of vortex generators in the flow. Percy and Stuart, (Ref. 16), extended the study of the effects of various design parameters and concluded that the strength and disposition of the individual induced vortices was more important than the details of the boundary layer upstream of the imposed pressure gradient. These results suggest that modeling of the vortex generators within a marching RNS analysis could be be very successful, probably because of the relative length scales encountered between the vortex generator region and the inlet duct itself.

The model for the vortex generators within the RNS analysis, described by Kunik, (Ref. 17), takes advantage of the stream function-vorticity formulation of the governing equations. The shed vortex is modeled by introducing a source term into the vorticity equation that is a function of the geometric characteristics of the generators themselves. This source term is introduced at every point in the cross-plane in the form of the following expression

$$
\Gamma_{p}=\Gamma_{0} e^{-\left(c_{1} r^{2}\right)}
$$

where $\Gamma_{p}$ is the vortex strength at any point in the crossplane, $\Gamma_{0}$ is the vortex strength at the tip of the generator, $r$ is the distance between the field point and at the tip of the generator, and $c_{1}$ is a constant which controls the decay of the shed vortex strength in the crossplane. This vortex

model resembles the one proposed by Squire, Ref. 18, except that it neglects the variation of viscosity in the crossplane.

\section{Inlet Duct Geometry and Flow Descriptors}

The reference line or centerline, in addition to defining a coordinate system in which to make the calculations, also characterizes the inlet duct in terms of a parameter $\tau$. Thus, the duct contours can be represented in terms of the centerline space curve

$$
\begin{aligned}
X_{c l} & =X(\tau) \\
Y_{c l} & =Y(\tau) \\
Z_{c l} & =Z(\tau)
\end{aligned}
$$

with prescribed cross-sectional planes normal to the centerline with flow area $A(\tau)$, and a geometric shape factor $S F(\tau)$ defined by

$$
S F=1.0+\frac{2}{D_{h y d} A} \int_{0}^{2 \pi} a b s\left[r-\frac{D_{h y d}}{2}\right] r^{2} d \theta
$$

which characterizes the cross-sectional shape of the duct. In equation (5.4) $D_{\text {hyd }}$ is the local hydraulic diameter, A the local flow area, and $r(\theta)$ is the local cross- sectional radius of the inlet duct in the local transverse plane. As the cross sectional shape approaches a circle, $S F$ will approach the value of 1.0 .

There are a number of flow discriptors that will be use to characterized the effects of inflow distortion and its transmission through the inlet duct. These include the static pressure coefficient and the mass flow averaged static pressure coefficient defined by

$$
C p=\frac{\left(p-p_{0}\right)}{q_{0}}
$$




$$
\overline{C_{p}}=\frac{1}{\dot{m}} \int_{A} \rho u_{p}\left[C_{p}\right] d A
$$

where $m$ is the mass flow given by

$$
\dot{m}=\int_{A} \rho u_{p} d A
$$

and $p_{0}$ and $q_{0}$ are the reference static and dynamic pressure. Likewise, the total pressure coefficient and the mass flow average total pressure are defined by

$$
\begin{gathered}
C p_{t}=\frac{\left(P t-p_{0}\right)}{q_{0}} \\
\overline{P t}=\frac{1}{\dot{m}} \int_{A} \rho u_{p}(P t) d A
\end{gathered}
$$

Thus, the mass flow averaged inlet total pressure recovery $\pi_{d}$ is given by

$$
\pi_{d}=\frac{\overline{P t}_{e f}}{\overline{P t_{i}}}
$$

where the subscript (ef) refer to the engine face station or aerodynamic interface plane, and $(i)$ indicates the inflow station.

In order to quantify effects of vortex disturbances entering the inlet, a number of parameters will be defined to characterized this type of disturbance. These parameters include the secondary flow kinetic energy

$$
K E_{s}=\int_{A} \rho u_{p}\left[\frac{\left(v_{s}^{2}+w_{s}^{2}\right)}{2}\right] d A
$$

the local swirl kinetic energy

$$
K E_{\theta}=\int_{A} \rho u_{p}\left[\frac{\left(v_{\theta}^{2}\right)}{2}\right] d A
$$

and the local primary flow kinetic energy

$$
K E_{p}=\int_{A} \rho u_{p}\left(\frac{u_{p}^{2}}{2}\right) d A
$$

The ratio of local secondary to primary flow kinetic energy becomes

$$
\kappa_{s}=\frac{K E_{s}}{K E_{p}}
$$

and the ratio of swirl to primary flow kinetic energy is given by

$$
\kappa_{\theta}=\frac{K E_{\theta}}{K E_{p}}
$$




\section{Steady State Engine Face Distortion Descriptors}

It is impractical to measure anything at the engine face when the engine is installed and operating, consequently, the engine and inlet designers agreed upon an Aerodynamic Interface Plane (AIP) which is forward of the compressor face but sufficiently close to the engine face to have a similar flow field. Current U.S. practice uses forty or forty eight transducer probes arranged in eight rakes with five or six rings. The radius of each ring is set such that all probes are at the centroid of equal areas. All distorton descriptors, whether they quantify steady state or transient distortion conditions, are always calculated relative to the standard rake located at the AIP.

The most widespread quantitative distortion descriptor available in the literature, because of its use in the earliest measurements on inlet ducts in the late 1950's, is simply:

$$
D t=\left(\frac{P t_{\max }-P t_{\min }}{P t_{\text {ave }}}\right)
$$

where $P t_{\text {max }}$ is the maximum rake total pressure, $P t_{\min }$ is the minimum rake total pressure, and $P t_{\text {ave }}$ is the area weighted average rake total pressure. In experimental data reduction, it is assumed that the both the static pressure and temperature are constant and steady across the aerodynamic interface plane (AIP); thus both the velocity and Mach number can be considered functions only of total pressue and the distribution of this quantity is the only measurement that needs to be made. This parameter is always useful to determine for comparison purposes and to describe the 'general health' of inlet ducts irrespective of the type of powerplant that may be used.

More advanced distortion descriptors, introduced in the late 1960's and 1970's, take into account the $D t$ distortion of each ring of total pressure measurements. Thus, the radial distortion $D t_{\text {rad }}$ is defined as

$$
D t_{\text {rad }}=\left(\frac{P t_{\max }-P t_{a v e}}{P t_{\max }}\right)_{\text {ring }}
$$

where $P t_{a v e}$ is the average total pressure for a given ring radius and $P t_{\max }$ is the maximun local ring total pressure. The circumferential distortion $D t_{\text {cir }}$ is defined as:

$$
D t_{c i r}=\left(\frac{P t_{a v e}-P t_{\min }}{P t_{a v e}}\right)_{\text {ring }}
$$

where $P t_{\min }$ is the lowest average total pressure in any 60 -degree or 180 -degree arc for a given ring radius having an average ring total pressure $P t_{\text {ave }}$. 


\section{RESULTS AND DISCUSSIONS}

\section{Comparison and Validation with Experimental Data}

To demonstrate the accuracy of the numerical procedure obtained with the 3D RNS code for internal duct flows typical of high angle-of-attack conditions, a series of numerical simulations were carried out on the University of Tennessee diffusing S-duct validation case. Vakili, $\mathrm{Wu}$, Liver, and Bhat, Ref. 10, in this experimental investigation sponsored by NASA Lewis Research Center, obtained a series of measurements in a 30-30 degree diffusing S-duct of area ratio 1.5 with and without vortex generators, Figure 3. The 30-30 degree circular cross-section S-duct, shown in Figure 3, was made from two symmetric sections. The inside hydraulic duct diameter $D_{i}$ was $16.51 \mathrm{~cm}$. and the mean radius of curvature $\mathrm{R}$ was $83.82 \mathrm{~cm}$. A $72.20 \mathrm{~cm}$. straight pipe was installed upstream of the curved section to allow for the development of the turbulent boundary layer to the desired thickness. Another pipe of length $152.40 \mathrm{~cm}$. was installed downstream of the S-duct.

All measurements were made at an inlet Mach number of 0.60 at the reference measurement station in the straight section $\mathrm{X}=24.8 \mathrm{~cm}$. The flow parameters at this station were used as reference conditions for non-dimensionalizing the experimental data. The experimental survey stations correspond to $\mathrm{X} / D_{i}=0.0,1.29,2.49$, and 5.2. At each survey station, a five port cone probe was traversed radially at ten azimuthal angles, approximately 20 degrees appart, on both sides of the symmetry plane. At least seventy points were measured at each traverse.

A polar grid topology, (Figure 4), was chosen for the analysis of the University of Tennessee diffusing S-duct, consisting of 49 radial, 49 circumferential, and 101 streamwise nodal points in the half-plane. The internal grid was constructed such that the transverse computational plane was perpendicular to the duct centerline. Grid clustering was used both in the radial and circumferential directions by redistributing the nodal points along these coordinate lines to resolve the high shear region near the wall and the separation region in the second bend. The flow in the inlet duct was turbulent, with an entrance Mach number of 0.6 , Reynolds number based on hydraulic diameter of $1.76 \times 10^{6}$, and the inflow corresponds to a shear layer approximately $\delta / D_{i}=0.05$. These initial conditions were applied at approximately an axial station 1.54 inlet diameters $\left(D_{i}\right)$ upstream of the duct entrance.

Figure 5 shows a comparison between the experimental and computed total pressure coefficient contours at $X / D_{i}=5.2$. In both the experiment and analysis, the flow in the S-duct separated and reattached in the second bend upstream of $\mathrm{X} / D_{i}=5.2$. This was due both to the adverse pressure gradients and the effect of pressure-driven secondary flow resulting from duct curvature. Experimental measurements and computational results from the 3D RNS code include the phenomena of separation and reattachment upstream of X/Di $=5.2$ and show excellent agreement for a simple mixing length turbulence model.

As separation was encountered in the second bend of the S-duct, three pairs of vortex generator devices were installed in the duct at $\mathrm{X} / D_{i}=0.09$, and at azimuthal angles of $-38.0,0.0$, and 38.0 degrees relative to the top of the inlet, and with respect to the streamwise direction. The vortex generator pairs had geometric incidence angles of $+16.0^{\circ}$ and $-16.0^{\circ}$ relative to the duct centerline. Figure 6 shows the comparison between the experimental and computed total pressure coefficient contours at $\mathrm{X} / D_{i}=5.2$. Comparison of contour levels between the separated case, Figure 5, and the vortex generator case, Figure 6, show that the vortex generators successfully mixed the high energy core flow with the low energy flow in the wall region to supress separation. In general, the interaction between the induced vortex generator flow and the pressure driven secondary flow that was computed was physically realistic and the agreement between experiment and analysis is considered very good. 
Figures 7 and 8 show additional flow characteristics obtained with the 3D RNS analysis with the vortex generator modeling. The secondary flow structure from the vortex generator model just downstream of the generator region, i.e. at $X / D_{i}=0.18$ is shown in Figure 7 , and clearly reveals the three pairs of vorticies that arise from the three pairs of counter-rotating generators. The limiting streamline signature shown in Figure 8 indicates that the generator configuration tested elliminated the flow separation encountered in the second bend, and reveals the familiar topographical pattern through the generator region itself.

\section{Flow Separation and Vortex Liftoff}

The three dimensional separation encountered in the University of Tennesse diffusing Sduct was very large in area, Figures 9 and 10, but thin in the direction normal to the wall such that it did not alter the pressure distribution in a substantial manner. Secondary flow resulting from duct curvature caused an accumulation of boundary layer near the innerwall of the first 30 degree bend. The thick boundary layer thus established was especially susceptible to flow separation because of the adverse pressure gradients induced by the reverse curvature section of the second 30 degree bend. A comparison between the computed oil flow patterns (represented by the limiting streamline topology in figure 9) and the experimental oil flow patterns presented in figure 10 shows excellent correspondence. Of importance is the fact that the viscous marching RNS analysis method using FLARE approximations captures the reverse flow region of this separation in a single pass calculation.

To meet the required confidence level of code validation, it is also essential that the $3 \mathrm{D}$ RNS marching analysis be able to capture the known topological structure of the limiting streamlines in the vicinity of separation. The very important and striking features of the symmetric pair of spiral nodes and saddle points were clearly captured by the Reduced Navier Stokes analysis. This very familiar topological pattern is known to describe the important stage in the development of the pair of counter rotating vorticies that form in the first 30 degrees of turning that results in vortex liftoff in the second bend, Figures 9 and 10 . Vortex liftoff and the resulting separation are jointly a major cause of total pressure loss and distortion at the engine face.

In general, the flow separation studied here was dominated by pressure forces rather than shear forces, as would be the case with massive separation in the inlet duct, such that the influence of the stress-driven flows was small and the effective viscosity approach surprisingly successful. These separation interactions could be called "vorticity separation" since they are dominated primarily by the transport of vorticity through the inlet duct (see following section). The case with vortex generators was also dominated by pressure driven secondary flows, as testified by the very good results from a simple modeling of the vortex generators in terms of a vorticity signature calculated from the physical properties of the generators themselves. The class of problems dominated by pressure forces rather than shear forces are known as vorticity dominated flows, and make up a very important class of internal flow problems.

\section{Vorticity Dominated Internal Flow Fields}

A predominant direction of flow can be identified in many high Reynolds number flow problems, both externaly and internaly. This primary direction may be derived from the direction of flight in external aerodynamics or from the ducted nature of internal flows. Viscous effects are very important, but they are generally limited to the region near the wall. In curved ducts, the outer portions of the boundary layer away from the duct walls behave as a rotational inviscid flow region in which secondary flow is generated (in the terminology of secondary flow theory) by turning of the transverse shear. This rotational inviscid behavior is reminiscent of the middle rotational inviscid layer of triple-deck theory for a two-dimensional boundary layer subjected to a streamwise disturbance, although the latter case does not involve streamwise vorticity. As the flow proceeds downstream, the secondary flow convects the streamwise vorticity and distorts the primary flow in what amounts to a strong interaction in the transverse directions between viscous 
and inviscid (rotational or potential) flow regions. Significant regions of rotational but essentially inviscid flow occur as a result of this process, and the potential-core region will eventually disappear.

Several numerical and physical factors can affect accurate predictions of high Reynolds number vorticity-dominated internal flows. These include the effects of numerical smoothing, (in the case of Full Navier Stokes analyses), the turbulence model, and the need for sufficient fine grids to resolve the details of the viscous boundary layer in the near wall region where the vorticity is the largest. For the computations of the flow within the University of Tennessee diffusing S-duct, two calculations were made to study the effects of near wall grid resolution. In the first case, the radial grid spacing was chosen to give a nominal $y+$ value of 8.5 at the first grid point above the duct wall, and in the second case, a nominal $y+$ value of 0.5 was chosen. Figures 11 and 12 show the effects of this near wall grid lattice resolution on the total pressure coefficient contours, (Figure 11), and the secondary flow structure, (Figure 12), both at the engine face station, $\mathrm{X} / D_{i}=5.2$. The very strong effect of near wall grid resolution on the structure and strength of the engine face flow results from the very nature of vorticity dominated internal flows. Since the largest value of vorticity occurs in the near wall region, and secondary flow is generated by turning of streamwise vorticity (shear), a very strong effect of near wall grid resolution was thus realized as stronger secondary flow (Figure 12), which had consequently an appreciable influence on the primary flow (Figure 11). This is really an inviscid rotational phenomenon rather than a viscous interaction, and indicates that secondary flow has its ultimate origin very near the wall. The influence of the turbulence model will thus also strongly influence the strength of secondary flow, but only as an invsicid rotational phenomena through the near wall vorticity distribution and not necessarily as part of the turbulent properties of the flow.

Figure 13 presents the limiting streamline signature associated with vortex liftoff obtained from the RNS code for the $y^{+}=0.5$ calculation, while Figure 14 shows the partical traces of vortex liftoff, again for the $y^{+}=0.5$, calculation for particles originating at $y / \delta_{i}=0.03$ It is quite apparent that fluid particles that originate well inside the entrance boundary layer can influence the core region of the engine face station.

\section{Fundamental Internal Vortex Interactions}

Unlike external aerodynamics where the two-dimensional flow assumption is valid over extended regions of the flow field, the internal flow problem is plagued with three-dimensional flow effects. When viscous effects or other sources of vorticity are present, three-dimensional flows differ fundamentally from their two-dimensional counterparts in that large secondary flows are generated by a deflection of the primary flow and/or other mechanisms. Secondary flow theory (reviewed by Horlock and Lakshminaryana, (Ref. 20), and Lakshminarayana and Horlock, (Ref. 21) affords considerable insight into the generation of secondary flow and establishes that large secondary flows can be generated by small deflection of vorticity or shear. The large secondary flows thus generated often exert an appreciable influence on the primary flow, and thus aerodynamic performance, viscous losses, and engine face distortion can be significantly affected.

The geometry of the bounding walls and flow area distribution are the primary drivers for 3D flow effects in internal passages. The development of secondary flows in transition ducts or curved ducts provide examples of 3D flows generated internally. The effect of the threedimensional flow field on vorticity dynamics is described by the phenomenon of vortex stretching (reviewed by Batchelor, Ref. 22 ). The term $\bar{\omega} \cdot \nabla \bar{U}$ in the vorticity dynamics equation, (see for example, reference 22 ), where $\bar{\omega}$ is the vorticity vector and $\bar{U}$ is the velocity vector, describes the time rate of change of vorticity due to "stretching" of the vortex filaments by the 3D flow field. This phenomenon, i.e. vortex stretching, is purely inviscid in origin.

Another fundamental internal interaction that can arise is when vorticity is introduced externally, i.e. either through a vortex ingestion or generated internally by means of vortex gen- 
erators. The resulting flow that develops under these circumstances has its origin in inviscid flow theory as well. Batchelor, (Ref. 22), describes the spiral motion of an initially straight streamwise vortex filament in a cylindrical duct with area contraction. The qualitative nature of this flow was such that as the vortex passed through the contraction section, it moved radially inward, and the azimuthal velocity of a material point on the vortex-line increased according to the rule $r \omega=$ const, hence it increased. Therefore, the predominant characteristic of vortex motion within an inclosed duct is the spiraling of the vortex core in a direction having the same sense as the vortex. Viscous effects are important, but their influence is felt by increasing boundary layer blockage thus causing the vortex-filament to move radial inward. The azimuthal velocity will increase as a result of $r \omega=$ const. behavior but decrease as a result of diffusion of vorticity from the vortex axis.

\section{Vortex Interactions within the F/A-18 Inlet Duct}

Two types of vortex-boundary layer interactions will be examined in this study. In the first case, vorticity is generated internally by means of one pair of counter-rotating vortex generators within the F/A-18 inlet duct. The purpose of these generators was to suppress flow separation that was likely to occure downstream of the a small "bump" within the duct, rather than to control engine face distortion. The second type of vortex-boundary layer interaction that was examined was under the condition where vorticity was externally introduced by means of an ingested vortex.

The F/A-18 inlet duct geometry definition used in this study is is shown in Figure 15, and the single block polar grid topology, with 49 radial, 98 circumferential, and 101 streamwise points within this inlet duct is presented in Figure 16. The computations were made at an inlet entrance Mach number of 0.6 , and Reynolds number of $8.0 \times 10^{5}$ based on the hydraulic inlet diameter $\left(D_{i}\right)$ , and inflow conditions that correspond to a shear layer thickness $\delta / D_{i}=0.056$.

The vortex generators used in this study were located at a streamwise station $\mathrm{X} / D_{i}=3.53$ upstream from the engine, and at an azmuthhal angle of 180.0 degrees relative to the inlet vertical axis, (Figure 17). All vortex generator dimensions, (Figure 17) are normalized in terms of the hydraulic inlet diameter $\left(D_{i}\right)$, which was 23.34 inches. The vortex generator incidence angles were +16.0 and -16.0 degrees with respect to the axial direction. Shown in Figures 18 and 19 are the limiting streamline signatures without and with vortex generators respectively. The characteristic vortex signature of the limiting streamline is through and downstream of the vortex generator reqion quite evident in Figure 19. An interesting study that should be made is to determine the effect of vortex generator flow alignment on aerodynamic effectiveness.

The second type of vortex-boundary layer interaction to be examined is under the condition where vorticity is introduced by means of a vortex ingestion. The nomenclature used for the constuction of the ingested vortex is shown in Figure 20 . Generic entrance conditions were established in each case by centering the core of the vortex of dimension $\left(r_{\text {cord }} / R_{i}\right)$ at $\left(r_{c} / R_{i}, \theta_{c}\right)$, where $\left(R_{i}\right)$ is the hydraulic inlet radius. It must be understood that the detailed inflow conditions of a real vortex ingestion is largly unknown and must be determined experimentally, however, this study seeks only to represent the starting inflow conditions in a generic sense such that studies can be made to relate inlet performance, i.e. inlet recovery, and engine face distortion, to the geometric properties of the duct. Tables I and II present the range of test cases considered for this study, which have been arranged in three groups. In the first group, (Table I), the vortex entry point was held fixed at an $\left(r_{c} / R_{i}, \theta_{c}\right)$ of 0.779 and $225.0^{\circ}$ while the swirl angle decreased from 0.0 to $-45.0^{\circ}$, i.e. (counter-clockwise). The second group of cases, (Table II), also kept the entry point fixed at an $\left(r_{c} / R_{i}, \theta_{c}\right)$ of 0.779 and $225.0^{\circ}$, while the swirl angle increased from 0.0 to $40.0^{\circ}$, i.e. (clockwise). In the third group of cases, the swirl angle was held fixed at $-30.0^{\circ}$, while the radial location of the vortex core $\left(r_{c} / R_{i}\right)$ was increased from 0.657 to 0.987 . 
Figures 21 and 22 show the inflow vortex structure at the entrance, (Figure 21), of the inlet duct $\mathrm{X} / D_{i}=0.0$, and that vortex structure which would be experienced at the engine face station, (Figure 22), at $\mathrm{X} / D_{i}=6.5$, for both the clockwise vortex and counter-clockwise vortex, with swirl angles of $+10.0^{\circ}$ and $-10.0^{\circ}$, respectively. The RNS solution within the F/A-18 inlet duct showing the limiting streamlines with an ingestion of $\mathrm{a}+10.0^{\circ}$ clockwise vortex is shown in Figure 23 , while the limiting streamline pattern produced by the of $-10.0^{\circ}$ counter-clockwise vortex is shown in Figure 24. In general, the entrance vortex wants to spiralal around the inside surface of the inlet duct in a direction corresponding to the sense of the initial vortex, i.e. whether the vortex is rotating clockwise or counter clockwise. This spiralaling characteristic around the inside wall of the inlet duct is indicated in Figures 23 and 24 by the coalescence of the limiting streamlines. This coalescences of the streamline reveals the vortex surface trajectory, and indicates a secondary (transverse) flow separation within the duct. The influence of the vortex generator can be seen for the clockwise vortex ingestion, (Figure 24), but interestingly, the counter clockwise vortex impinges on the vortex generators, deflecting the vortex trajectory, and rendering the generators ineffective.

In order to quantify the effects of a vortex disturbance entering the inlet duct, both the secondary to primary flow $\left(\kappa_{s}\right)$ and swirl to primary flow $\left(\kappa_{\theta}\right)$ kinetic energy ratio were defined by equations (5.14) and (5.15) respectively. Figures (25) and (26) presents both the swirl and secondary flow the kinetic energy signature of the $+10.0^{\circ}$ clockwise vortex ingestion, (Figure 25), and $-10.0^{\circ}$ counter-clockwise vortex ingestion, (Figure 26), both compared to the background signature, i.e. $\alpha_{s}=0.0^{\circ}$. It is assumed that the swirl kinetic energy component $\left(\kappa_{\theta}\right)$ is that portion of the secondary flow kinetic ratio $\left(\kappa_{s}\right)$ that would be elliminated with inlet guide vanes, (IGV's). IGV's are present in all American modern military aircraft high performance engines except the TF41, which is a derivative of the European Spey.

Thus, these parameters provide a unique signature which can track and quantify the passage of each and every vortex disturbance entering the inlet duct. Therefore, a relationship between the inlet duct geometry, described in terms of a distribution of area, $A(\tau)$, and shape factor, $S F(\tau)$, along a centerline space curve with geometric properties of slope and curvature, can be established. This provides a quantitative methodology whereby the aerodynamic flow quantities characterizing the vortex ingestion can be directly related to the important geometric quantities characterizing the inlet duct geometry.

Figures 27 through 30 present the particle traces released from $y / \delta_{i}=1.14$ for vortex ingestion swirl angles $\alpha_{s}=0.0^{\circ},-10.0^{\circ},-20.0^{\circ}$, and $-30.0^{\circ}$. The spiralling characteristics of the ingested vortex are clearly revealed in these figures along with the characteristic that as the initial swirl angle increases, the vortex helical angle increases, i.e. the vortex spiralls further around the inside surface of the duct. Particle traces resulting from an ingestion of a counter-clockwise vortex of strength $\alpha_{s}=-40.0^{\circ}$, (Figure 31), reveal very clearly the helical-like vortex trajectory as seen from a downstream view. Figure 32 presents the trajectory of the vortex center relative to the inlet duct centerline for the $\alpha_{s}=-40.0^{\circ}$ vortex ingestion case.

The effect of vortex strength on the engine face impingement location is shown in Figure 33. The $\alpha_{s}=0.0^{\circ}$ impingement location represents the ingested vortex entry point at the inlet face. Thus, as the strength of the ingested vortex increases, its compressor face angular impingement location increases and the core of the vortex moves radially inward. The radial inward movement is a results of increased boundary layer blockage caused by the increased strength of the ingested vortex. Figure 34 presents the effect of radial entry point on engine face vortex impingement location, i.e. the third set of vortex ingestion test cases listed in Table II. At a vortex entry position greater than $r_{c} / R_{i}=0.779$, the inflow vortex is "cut-off" by the lip, and consequently, its strength decreases, i.e. the circulation around any closed path surrounding the vortex filament decreases. As a consequence, a maximum angular impingement location occurs at the engine face station, (Figure 34). 
The overall qualitative nature of a vortex ingestion, at the Reynolds number considered, was basically "inviscid like", i.e. the dominant aerodynamic characteristics have their origin in inviscid flow theory. Therefore, the predominant characteristic of vortex motion within an inclosed duct is the spiralaling of the vortex core in a direction having the same sense as the vortex. Viscous effects are important, but its influence is felt by increasing boundary layer blockage, thus causing the vortex-filament to move radial inward. The azimuthal velocity will increase as a result of $r \omega=$ const. behavior but decrease as a result of diffusion of vorticity from the vortex axis. 


\section{CONCLUSIONS}

The goal of the present investigation was to combine the Reduced Navier Stokes solution technique with the concept of partitioned geometry and mesh generation to form an efficient 3D RNS code aimed at the CFD analysis-design engineering enviornment. Partitioned geometry and mesh generation is a geometry pre-processor to augment existing geometry and grid generation programs and has the advantage that the solver can (1) recluster an existing gridfile mesh lattice, and (2) perturb an existing gridfile geometry and mesh to alter the cross-sectional shape or centerline definition without returning to the external geometry and grid generator.

The present results provide a quantitative validation of the initial value space-marching $3 D$ RNS procedure and demonstrates accurate predictions of the engine face flow field, with a separation present in the inlet duct as well as when vortex generators are installed to supress separation. The computing time, CPU $=6.5 \mathrm{~min}$. on the CRAY XMP for $2.28 \times 10^{5}$ grid points, for both the baseline case and the case with three pairs of counter-rotating vortex generators, is sufficiently rapid for routine use in an analysis-design engineering environment.

Initial value space marching 3D RNS procedures using FLARE approximations can adequatly describe the topographical features of $3 \mathrm{D}$ flow separation associated with vortex liftoff within inlet ducts. The success of this RNS analysis in describing this phenomenon is due to the existence of a class of separated flows, which can be called "vorticity separations", which are dominated by the transport of vorticity rather than turbulent shear effects.

Adequate resolution of turbulent flows including viscous sublayer resolution down to a $y^{+}=0.5$ appears necessary to obtain accurate calculations on the strength of secondary flows that develop in typical inlet ducts, and in particular, the size of the separated region and the engine face or AIP distortion signature.

Selected use of vortex flow control to eliminate separation and improve the engine face distortion index can feasibly be analyzed using 3D RNS initial value procedures. The fundamental nature of secondary flow, which includes internal vortex interactions that arise either from a vortex ingestion or vortex generators, are primarily inviscid-rotational in character, hence pressure driven. Thus, accurate predictions of these fundamental interactions are possible using simple mixing length turbulence models.

The present investigation also demonstrates the ability of the three dimensional RNS techniques to analyze the flow physics associated with vortex ingestion in general geometries such as the F/A-18 inlet duct. Although no experminental validation data exsist on this phenomena, the overall correctness of the computed flow physics can be argued from simplified rotational inviscid flow theory. An overall computing time on the CRAY XMP of CPU $=14.5 \mathrm{~min}$. for $4.75 \times 10^{s}$ grid points makes this RNS computational approach ideally suited for routine use within an analysis design environment. 


\section{REFERENCES}

1. Advisory Group for Aerospace Research and Development (AGARD), "Engine Response to Distorted Inflow Conditions," AGARD CP-400, Sept., 1986.

2. Bowditch, D. N. and Coltrin, R. E., "A Survey of Inlet/Engine Distortion Compatability," AIAA-83-1166, June 1983.

3. Vatsa, V. N., and Wedan, B. W., "Development of an Efficient Multigrid Code for 3-D Navier Stokes Equations," AIAA Paper No. AIAA Paper No. 89-1791, June, 1989.

4. Briley, W. R. and McDonald, H., "Analysis and Computation of Viscous Subsonic Primary and Secondary Flow," AIAA Paper No. 79-1453.

5. Briley, W. R., and McDonald, H., "Three-Dimensional Viscous Flows with Large Secondary Velocities," Journal of Fluid Mechanics, March 1984, vol. 144, pp. 47-77.

6. Kreskovsky, J. P., Briley, W. R. and McDonald, H., "Prediction of Laminar and Turbulent Primary and Secondary Flows in Strongly Curved Ducts," NASA CR-3388, Feb. 1981

7. Levy, R., Briley, W. R., and McDonald, H., "Viscous Primary/Secondary Flow Analysis for Use with Nonorthogonal Coordinate Systems," AIAA Paper No. 83-0556, Jan. 1983.

8. Towne, C. E., and Anderson, B. H., "Numerical Simulation of Flows in Curved Diffusers with Cross-Sectional Transitioning Using a Three Dimensional Viscous Analysis," AIAA Paper No. 81-0003, Jan. 1981.

9. Towne, C. E., "Computation of Viscous Flow in Curved Ducts with Experimental Data," AIAA Paper No. 84-0531, AIAA 22th Aerospace Sciences Meeting, Jan. 1984.

10. Anderson, B. H., "Three Dimensional Viscous Design Methodology of Supersonic Inlet Systems for Advanced Technology Aircraft," Numerical Methods for Engine-Airframe Integration, American Institute of Aeronautics and Astronautics Series, 1986.

11. McDonald, H. and Camarata, F. J.: "An Extended Mixing Length Approach for Computing the Turbulent Boundary-Layer Development, Proceedings, Standford Conference of Turbulent Boundary Layers," Vol. I, Pub. by Stanford University, pp. 83-98, 1969.

12. Reyner, T. A. and Flugge-Lotz, I., "Interaction of a Shock Wave with a Laminar Boundary Layer," International Journal of Non-Linear Mechanics, Vol. 3, 1968.

13. Taylor, D., "Application of Vortex Generator Mixing Principile Principle to Diffusers," U.A.C. Rep. R-15064-5, Dec. 1948.

14. Valentine, E. F. and Carrol, R. B., "Effects of Several Arrangements of Rectangular Vortex Generators on the Static Pressure Rise Through a Short 2:1 Diffuser,"

NASA RM L50L04, Feb. 1951. 


\section{REFERENCES}

15. Valentine, E. F. and Carrol, R. B., "Effects of Some Primary Variables of Rectangular Vortex Generators on the Static Pressure Rise Through a Short Diffuser," NACA RM 52B13, May 1952.

16. Pearcy, H. H. and Stuart, C. M., "Methods of Boundary-Layer Control for Postponing and Alleviating Buffeting and other Effects of Shock-Induced Separation," Presented at the IAS National Summer Meeting, Los Angles, Calf., June, 1959.

17. Kunik, W. G., "Application of a Computational Model for Vortex Generators in Subsonic Internal Flows," AIAA Paper No. 86-1458, June 1986.

18. Squire, R., "Growth of a Vortex in Turbulent Flow," The Aeronautical Quarterly, Vol. 16, Pt. 3, August 1965, pp 302-306.

19. Vakili, A. D., Wu, J. M., Liver, P. A., and Bhat, M. K., "Experimental Investigation of Secondary Flows in a Diffusing S-Duct with Vortex Generators," NASA NAG3-233, July, 1986.

20. Horlock, J. H. and Lakshminarayana, B., "1973 Secondary Flows: Theory, Experiment, and Applications in Turbomachinery Aerodynamics," Ann. Rev. Fluid Mechanics, Vol 5, pp 247-280.

21. Lakshminarayana, B, and Horlock, J. H., "Generalized Expressons for Secondary Vorticity Using Intrinsic Coordinates," J. Fluid Mechanics. Vol. 59, pp. 97-115.

22. Batchelor, G. K., "An Introduction to Fluid Dynamics," Cambridge University Press, Cambridge, England, 1967. 


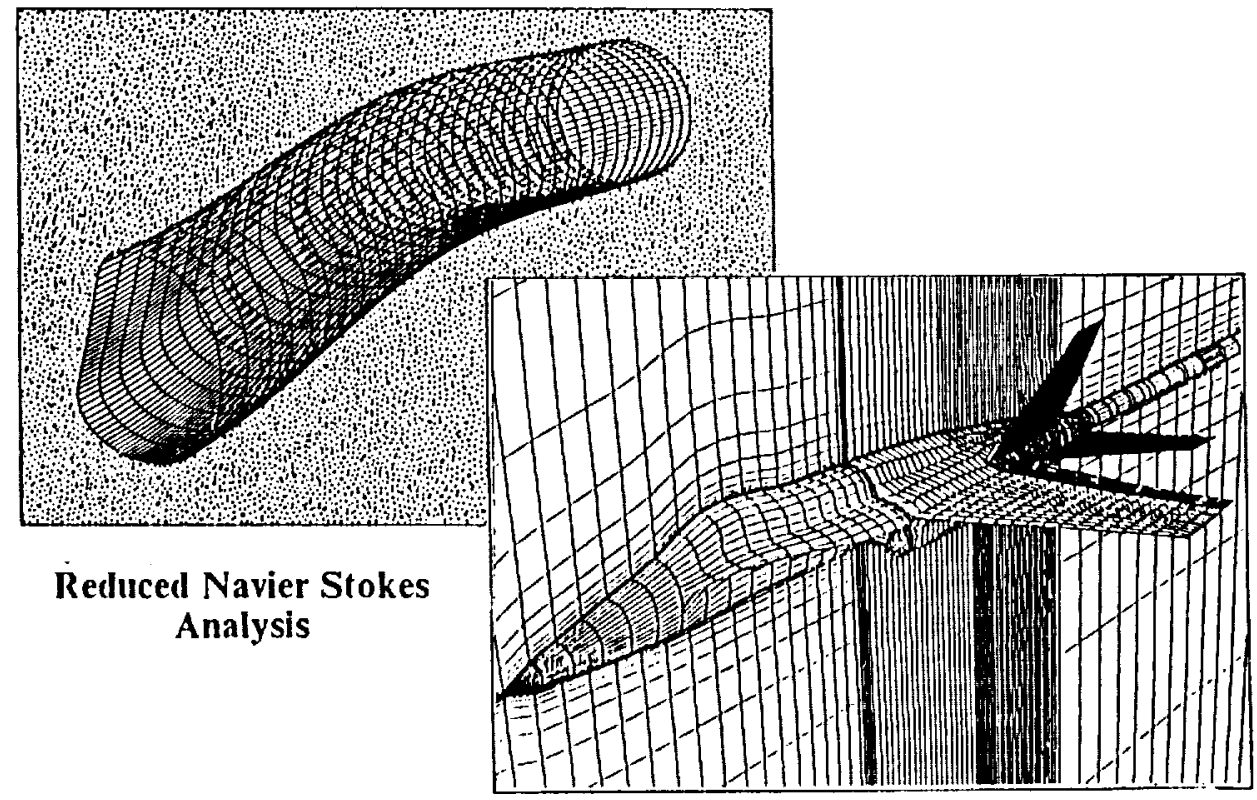

Full Navier Stokes Analysis

Figure (1) - Reduced Navier Stokes and Full Navier Stokes capabilities within an analysis-design engineering environment.

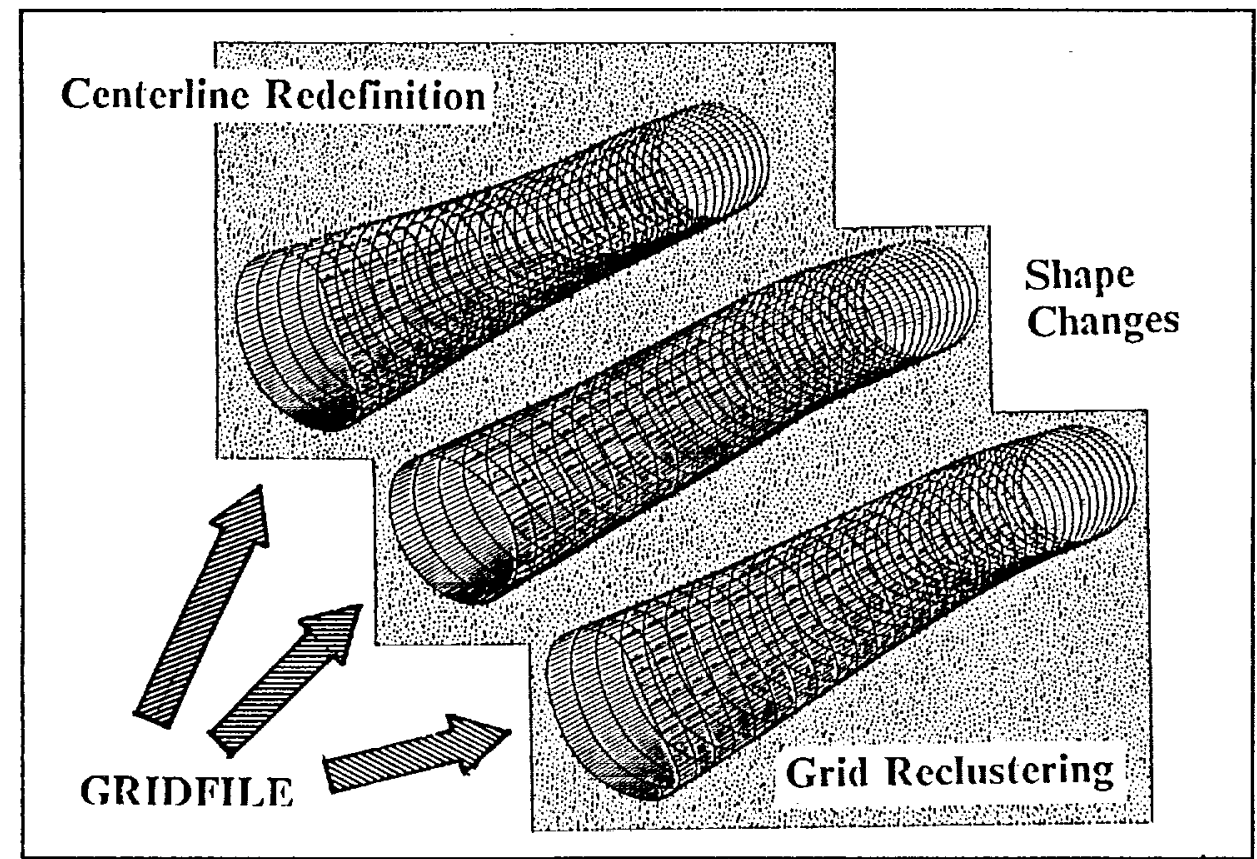

Figure (2) - Paritioned geometry and grid generation for inlet ducts within an analysis-design engineering environment. 


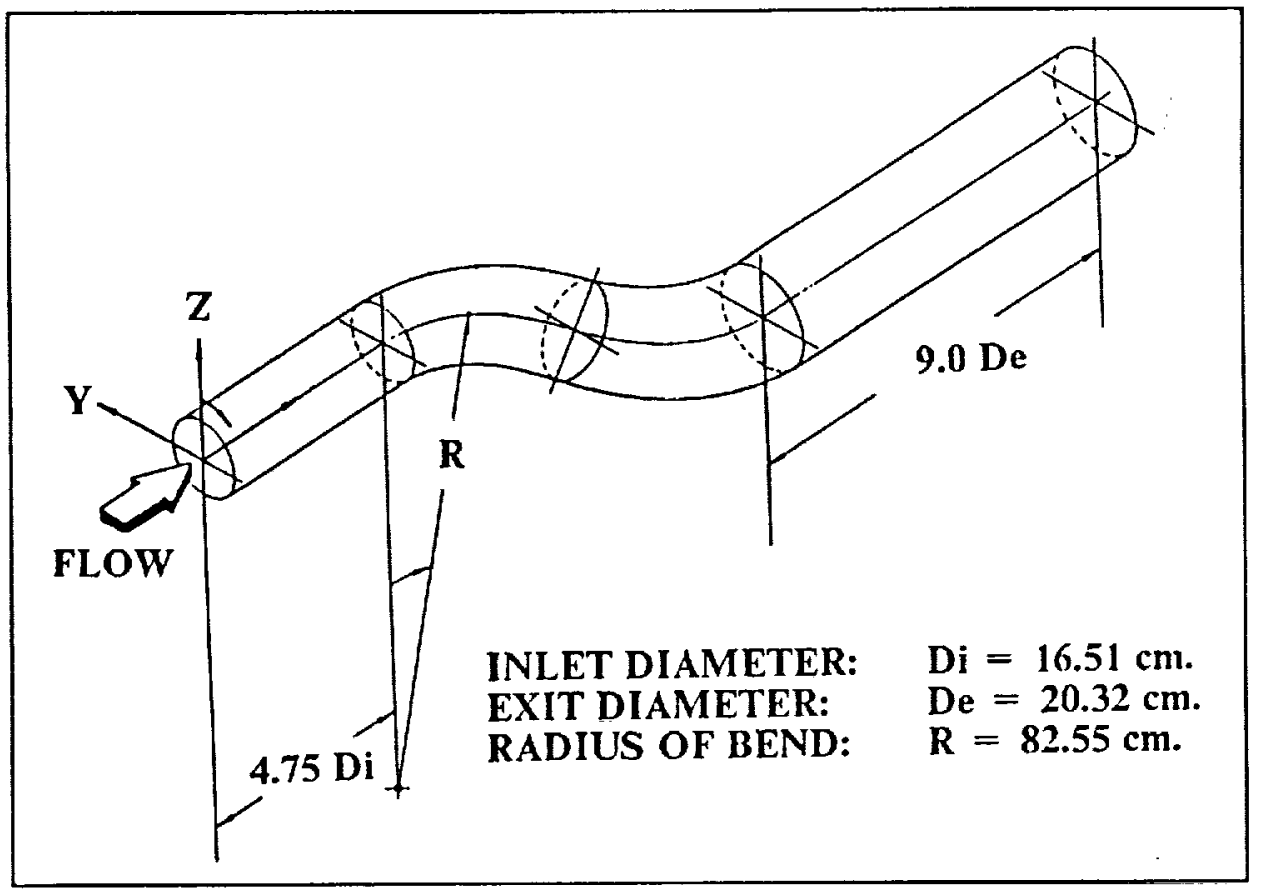

Figure (3) - Geometry definition of the Univ. Tennessee diffusing S-duct.

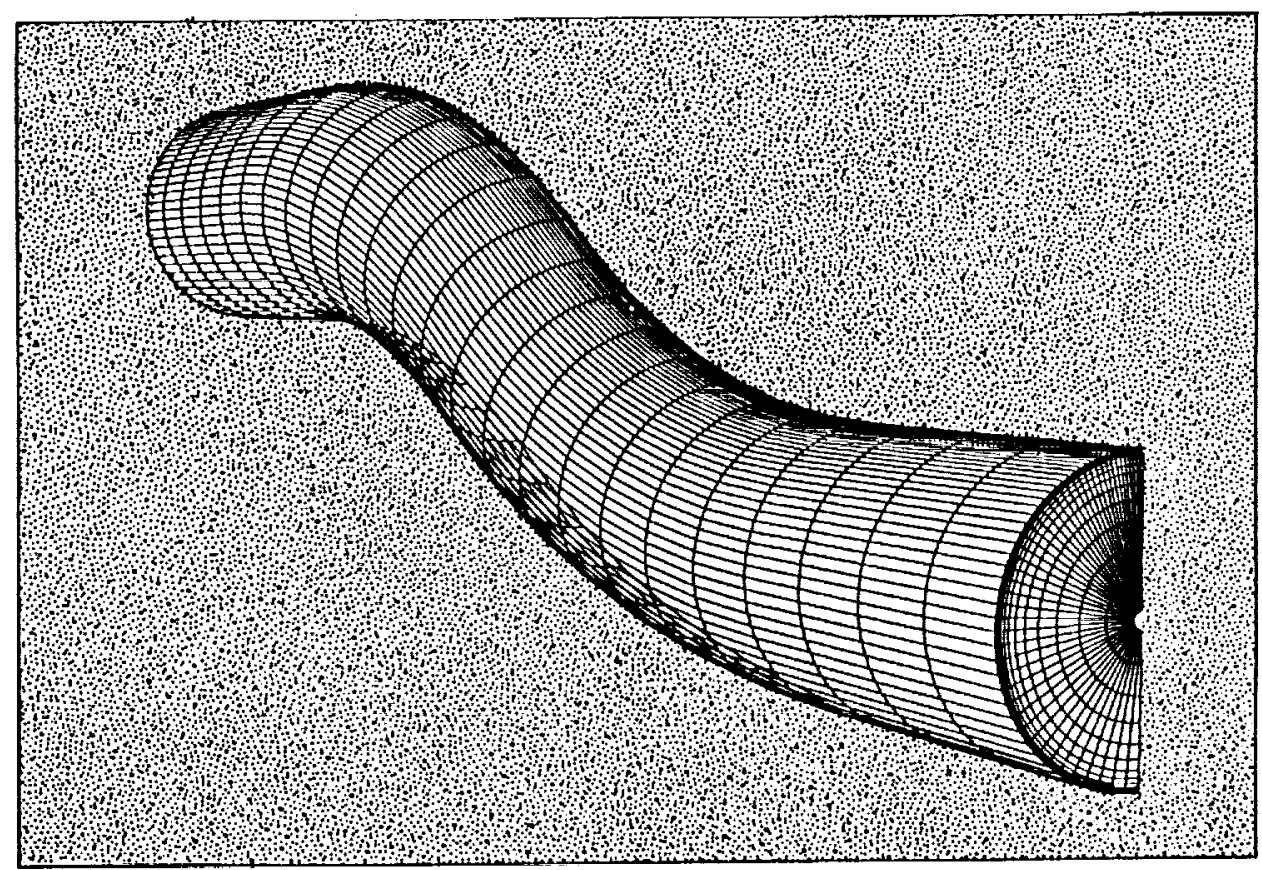

Figure (4) - Computation grid for the Univ. Tennessee diffusing S-duct. 


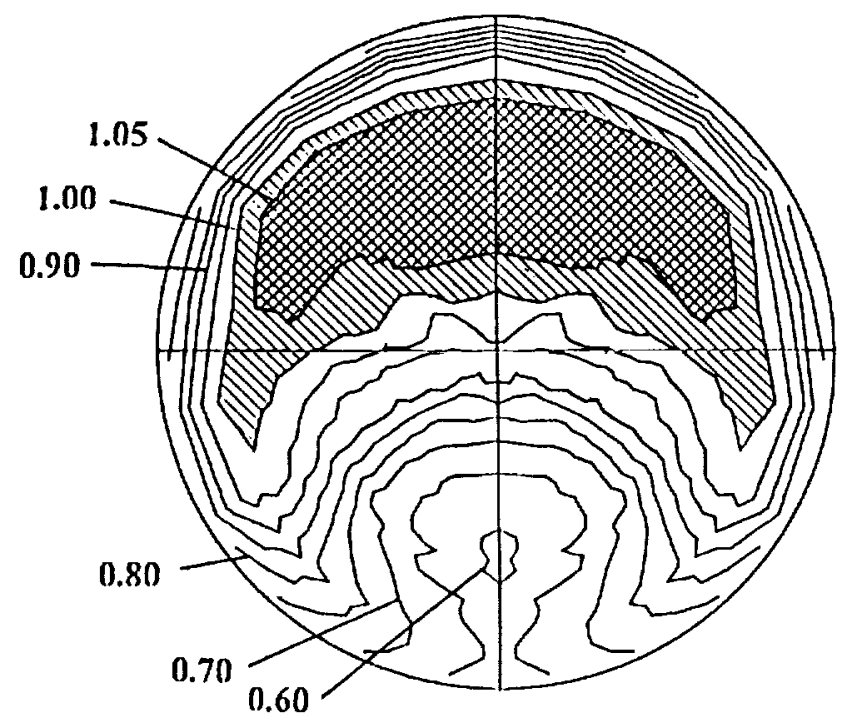

EXPERIMENTAL DATA

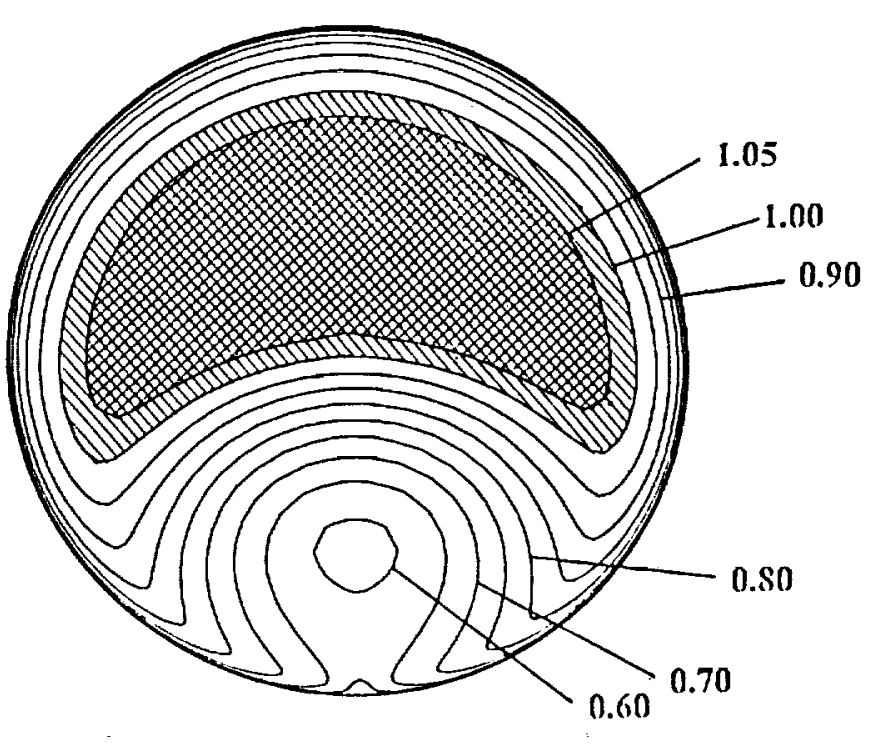

ANALYSIS, $y+=0.5$

Figure (5) - Total pressure coefficient contours without vortex generators, $\mathrm{X} / D_{i}=\mathbf{5 . 2}$.

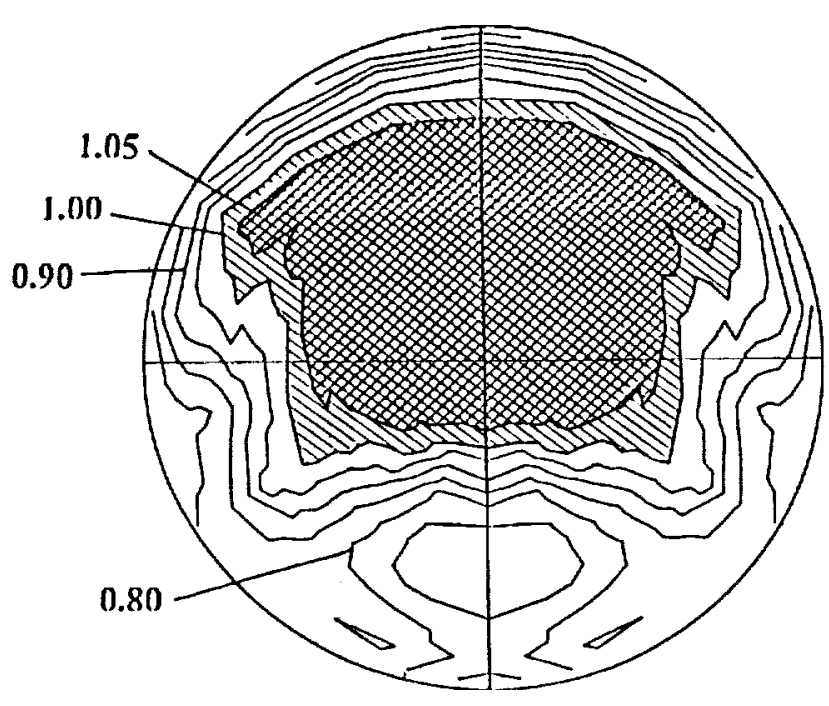

EXPERIMENTAL DATA

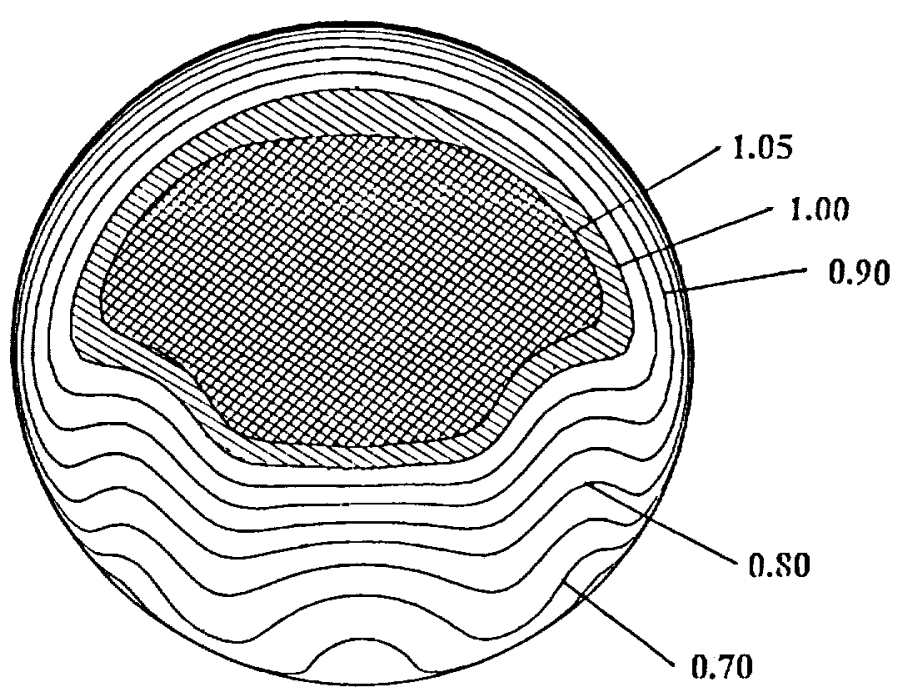

ANALYSIS,$y+=0.5$

Figure (6) - Total pressure coefficient contours with vortex generators, $\mathrm{X} / D_{i}=\mathbf{5 . 2}$. 


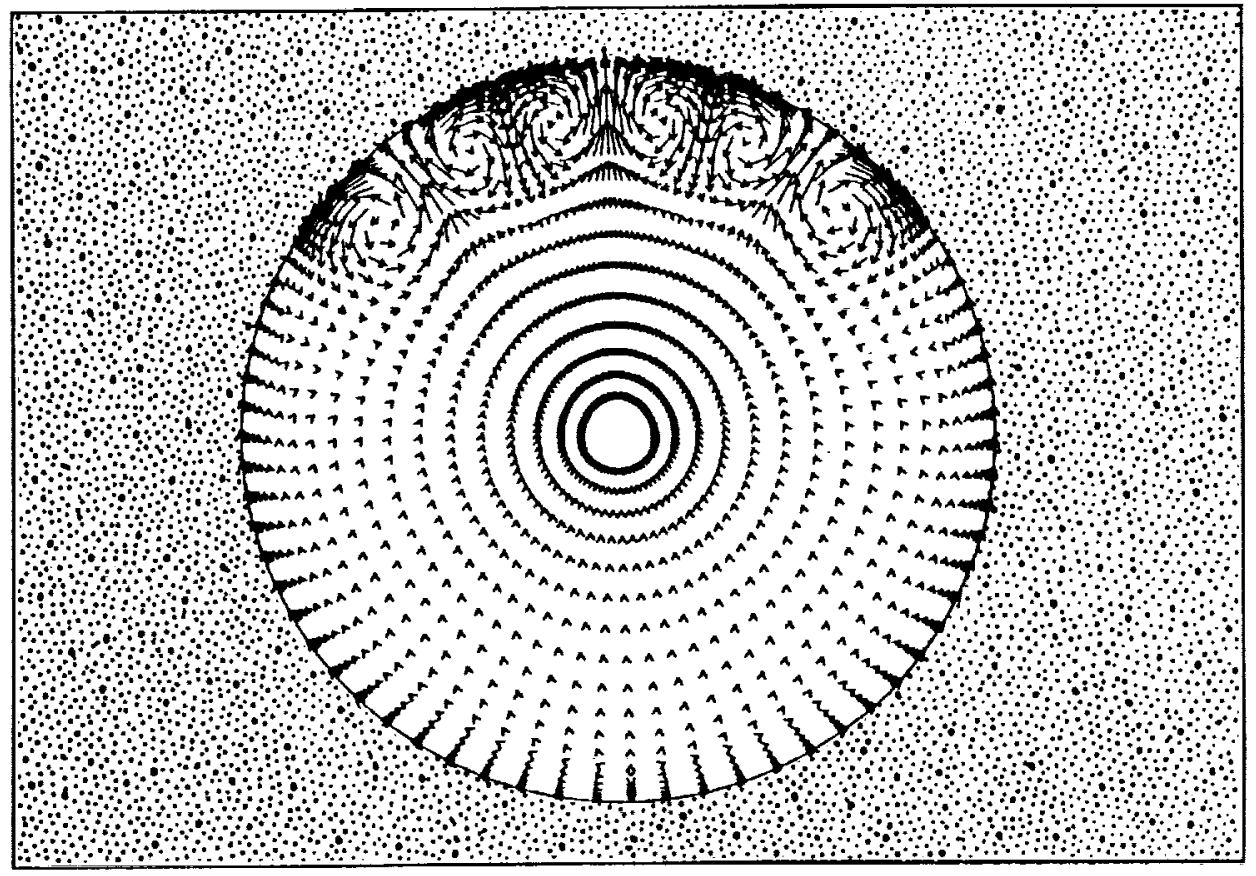

Figure (7) - Reduced Navier Stokes (RNS) solution showing secondary flow structure from vortex generator model, $\mathrm{X} / D_{i}=0.18$.

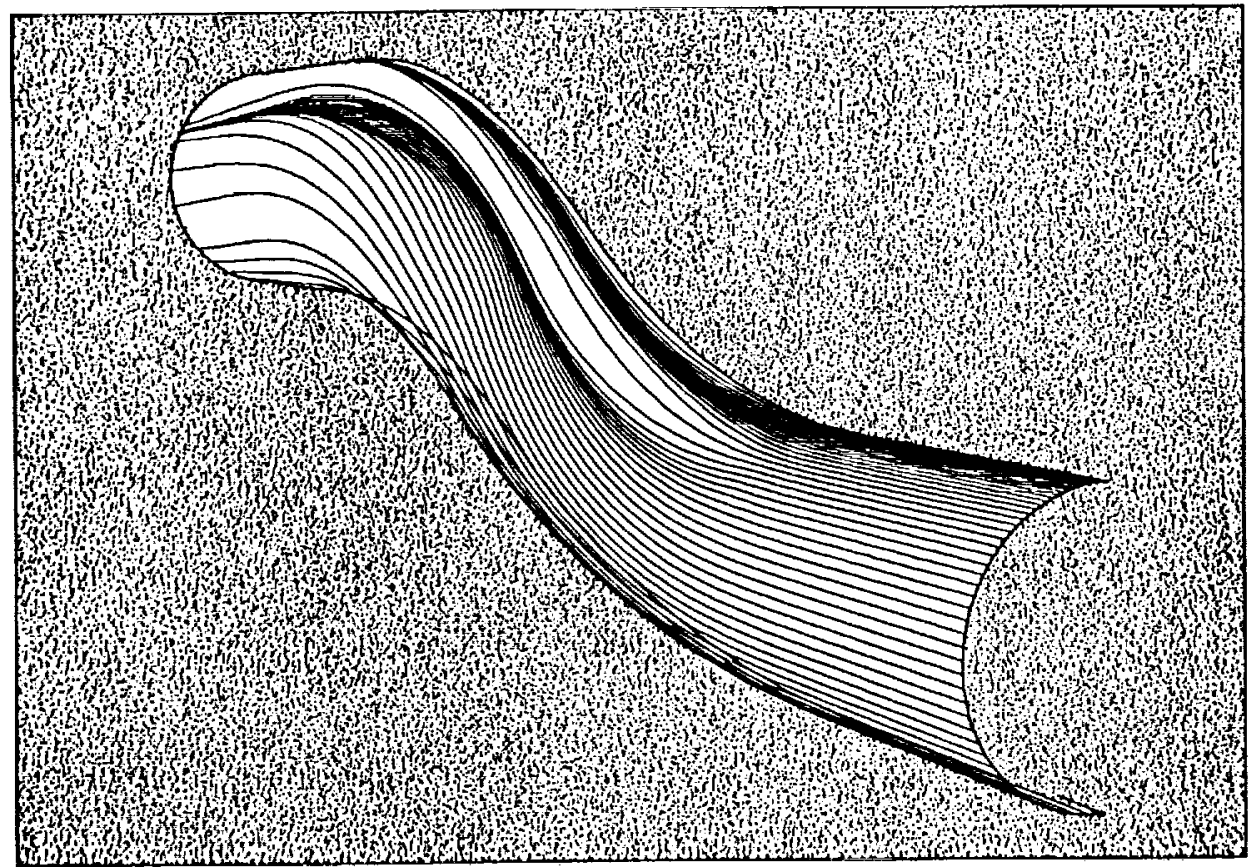

Figure (8) - Reduced Navier Stokes (RNS) solution showing limiting streamline signature of vortex generator region. 


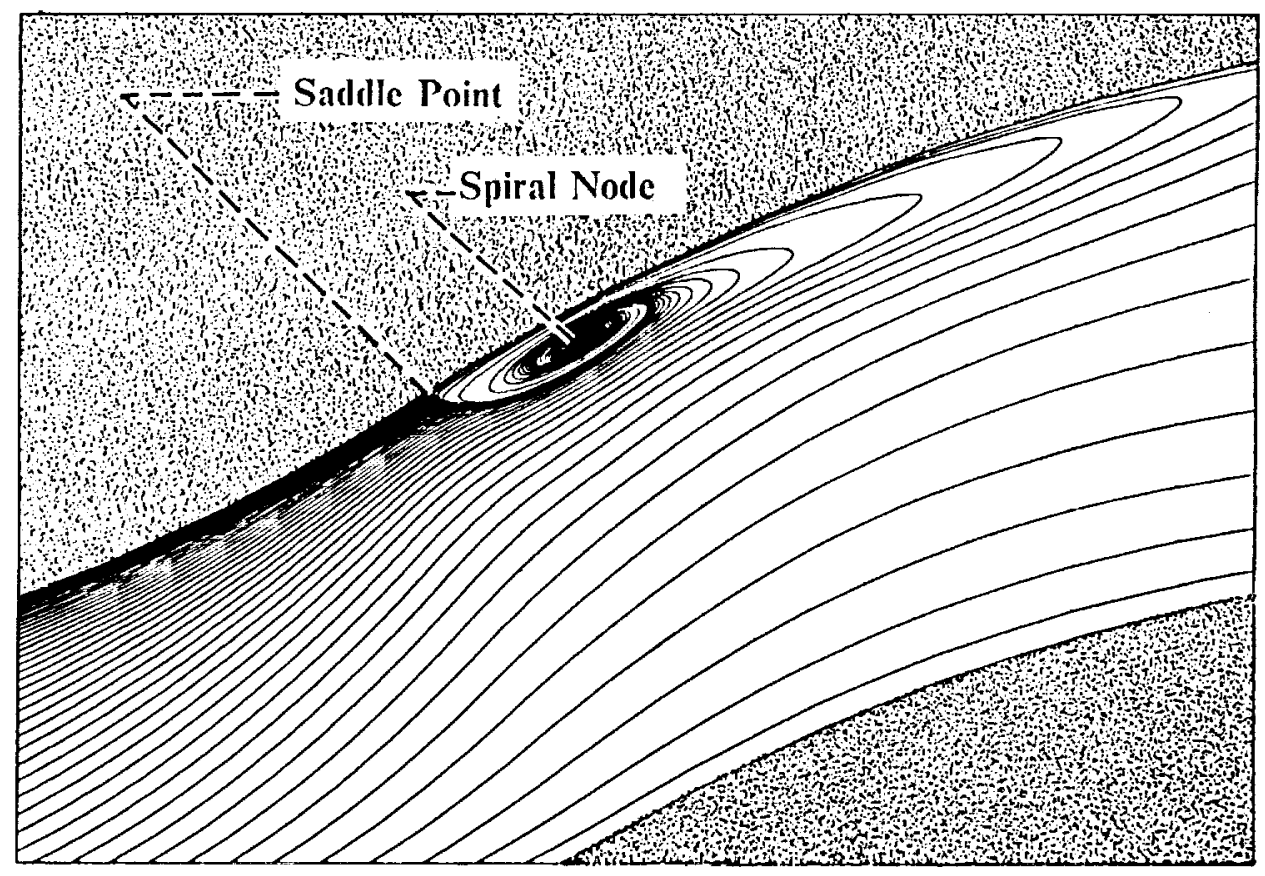

Figure (9) - Reduced Navier Stokes (RNS) solution showing limiting streamline signature of separation region within Univ. Tennessee diffusing $\mathbf{S}$-duct.

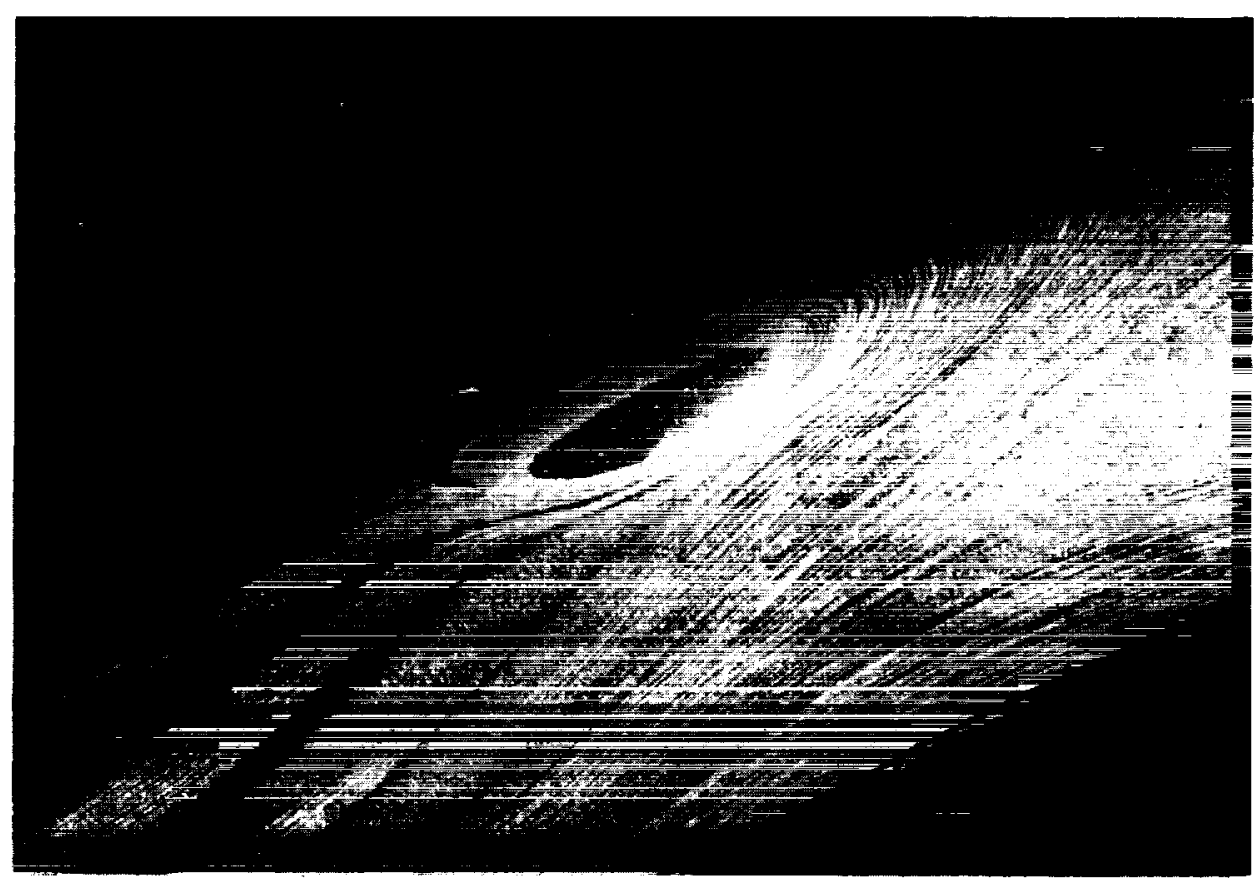

Figure (10) - Surface oil flow patterns showing separation region within Univ. Tennessee diffusing S-duct.

\author{
ORIGINAL FAGE \\ BLACK AND WHITE PHOTOGRAPH
}




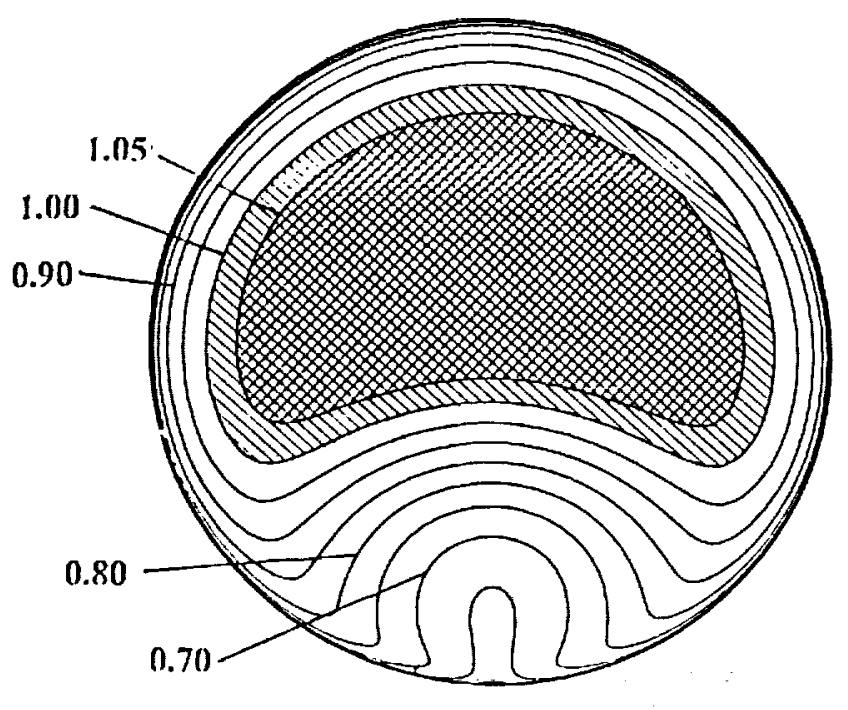

ANALYSIS, $\mathrm{y}+=8.5$

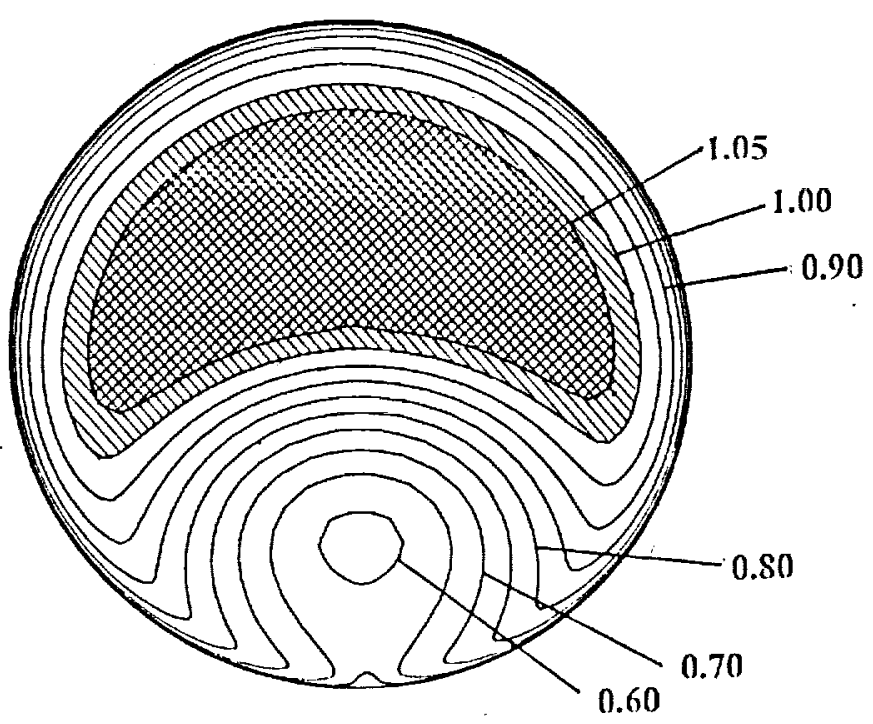

ANALYSIS, $\mathrm{y}+=0.5$

Figure (11) - Effect of near wall grid lattice resolution on total pressure coefficient contours, $\mathrm{X} / D_{i}=\mathbf{5 . 2}$.

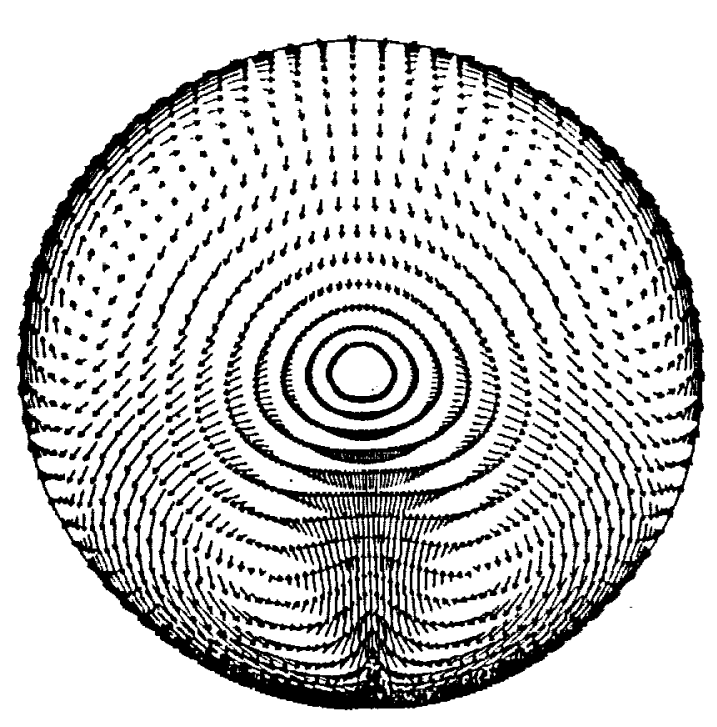

ANALYSIS, $y+=8.5$

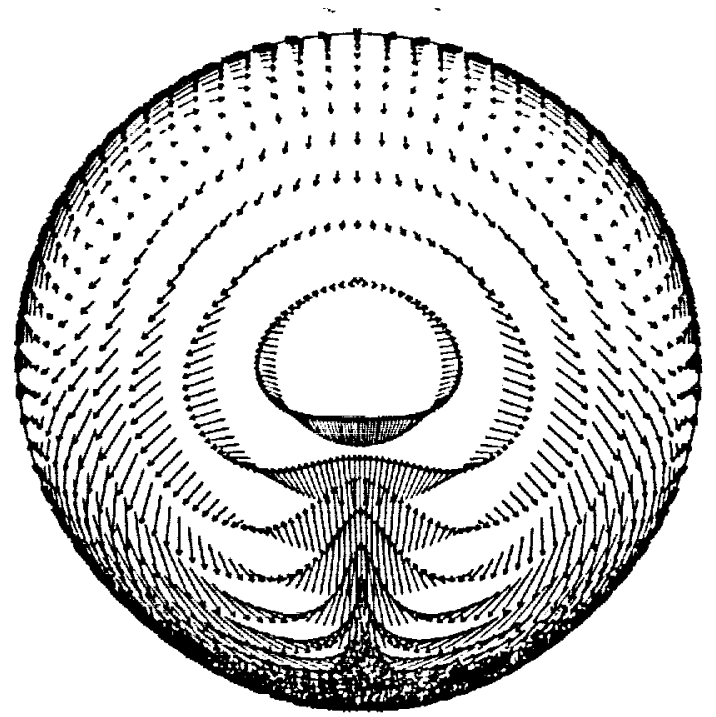

ANALYSIS, $y+=0.5$

Figure (12) - Effect of near wall grid lattice resolution on secondary flow structure, $\mathrm{X} / D_{i}=\mathbf{5 . 2}$. 


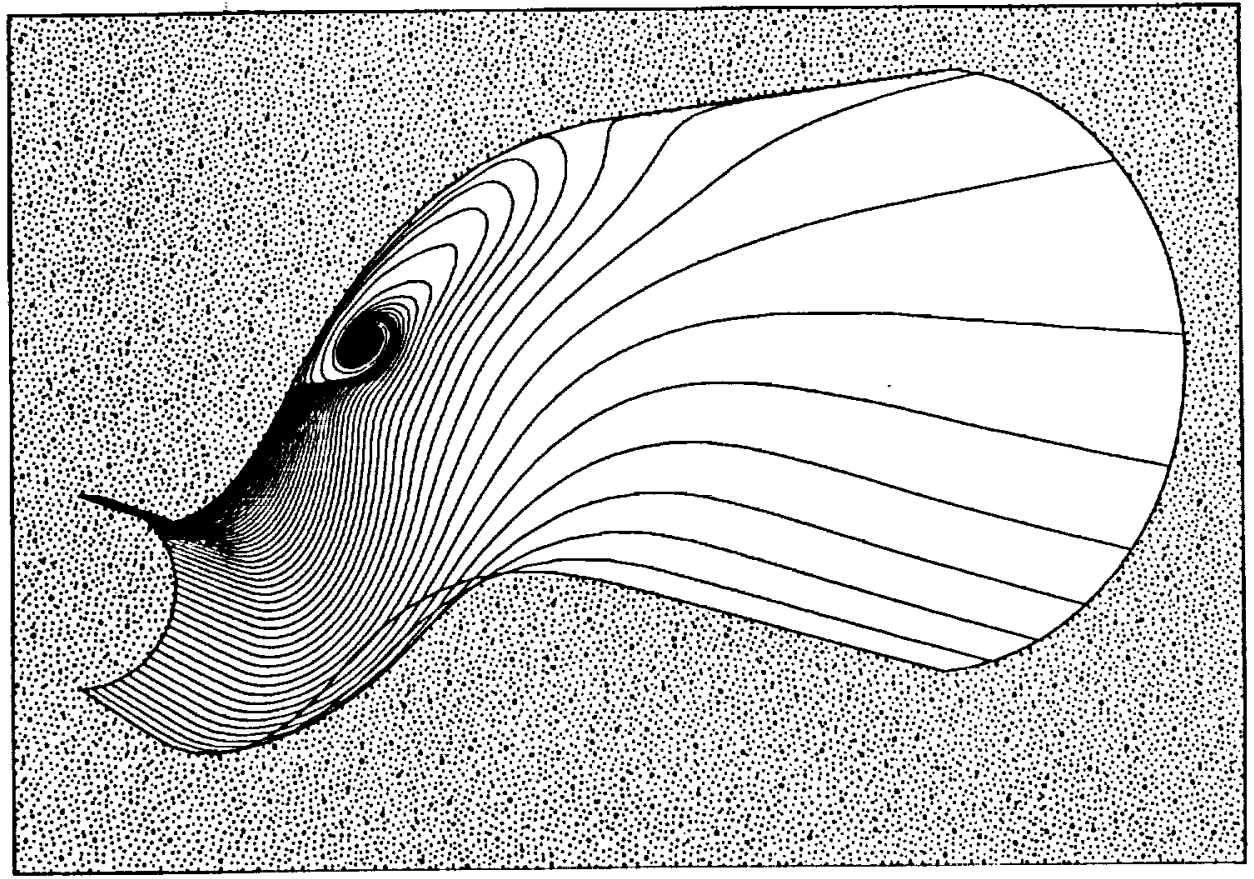

Figure (13) - Reduced Navier Stokes (RNS) solution showing limiting streamline signature of separation region.

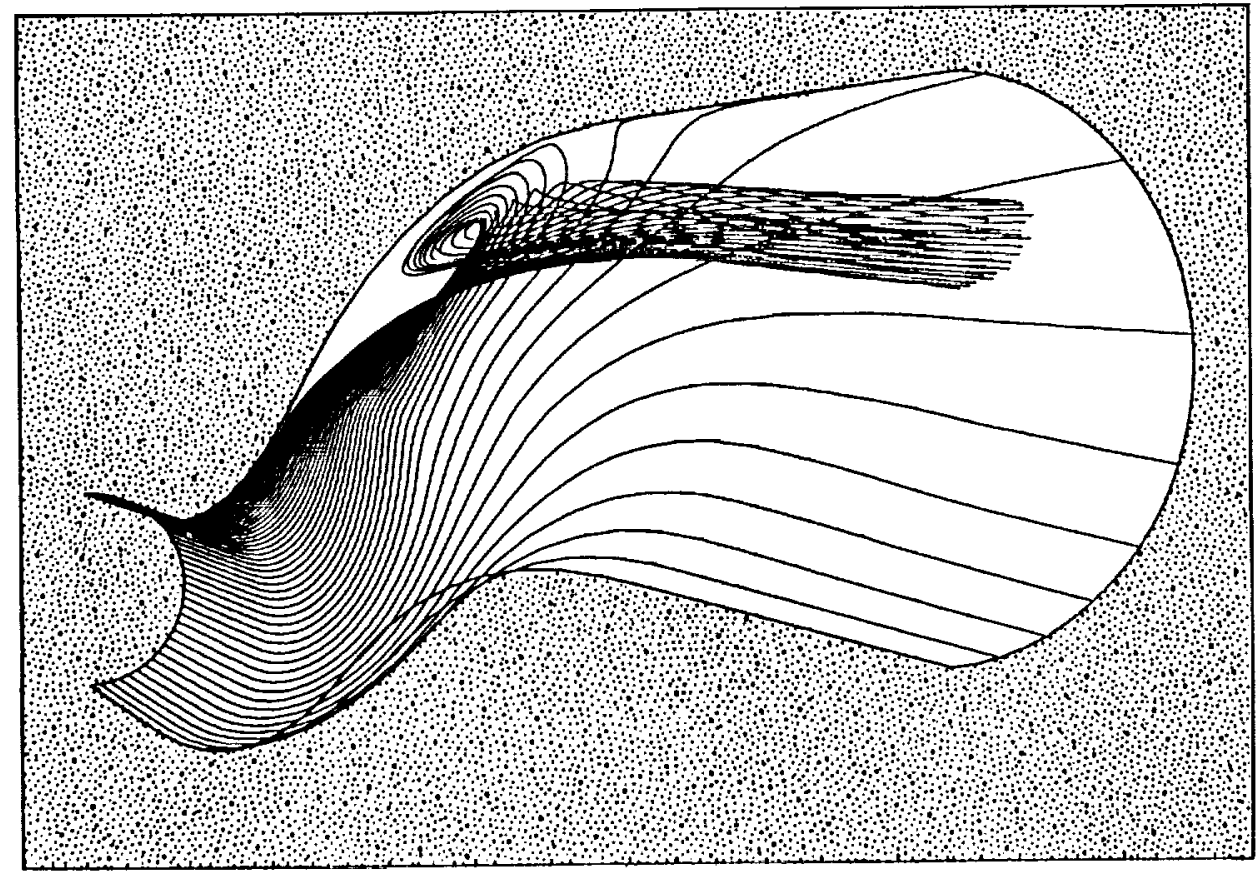

Figure (14) - Reduced Navier Stokes (RNS) solution showing particle traces associated with vortex liftoff, $y / \delta_{i}=0.03$. 


\section{ORIGINAL PAGE \\ BLACK AND WHITE PHOTOGRAPH}

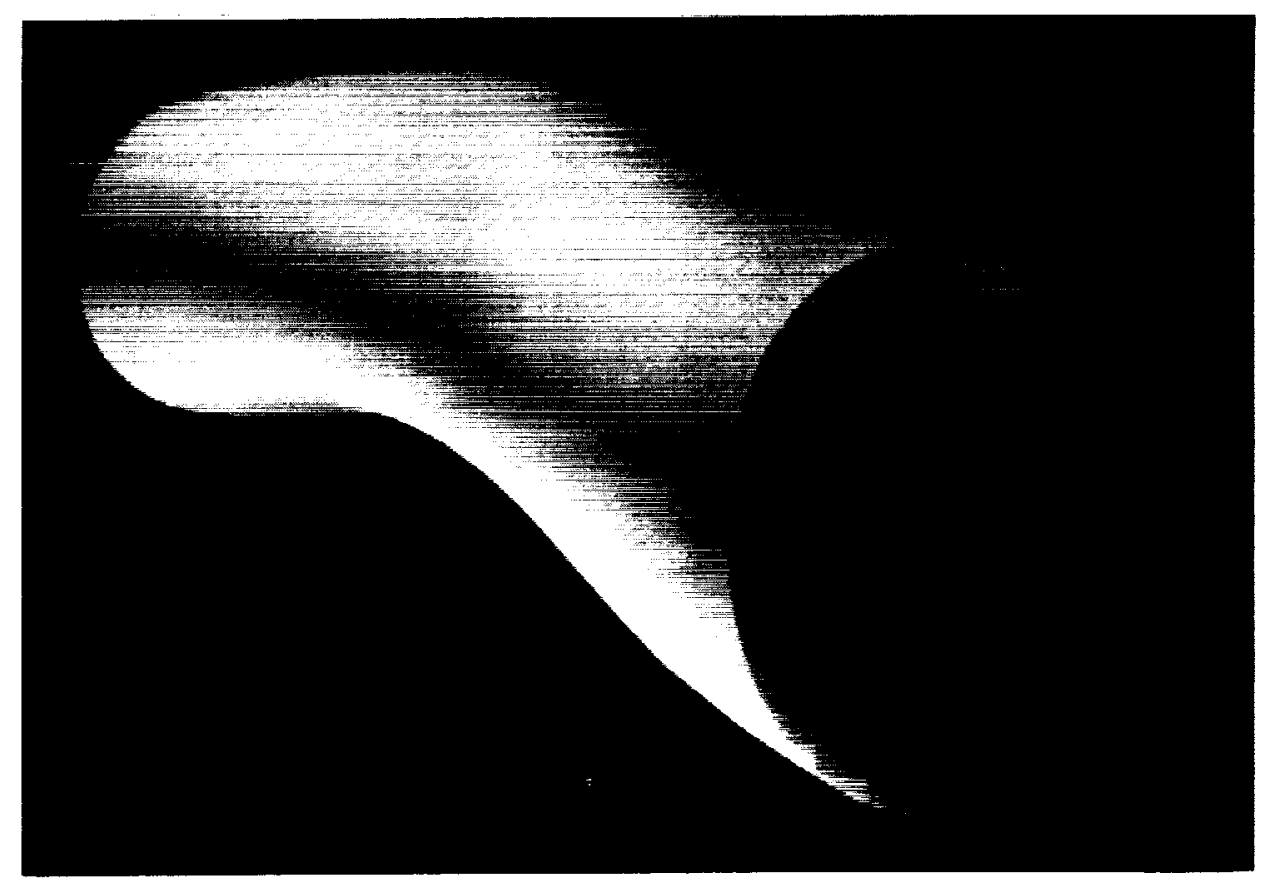

Figure (15) - Geometry definition of the F/A-18 inlet duct.

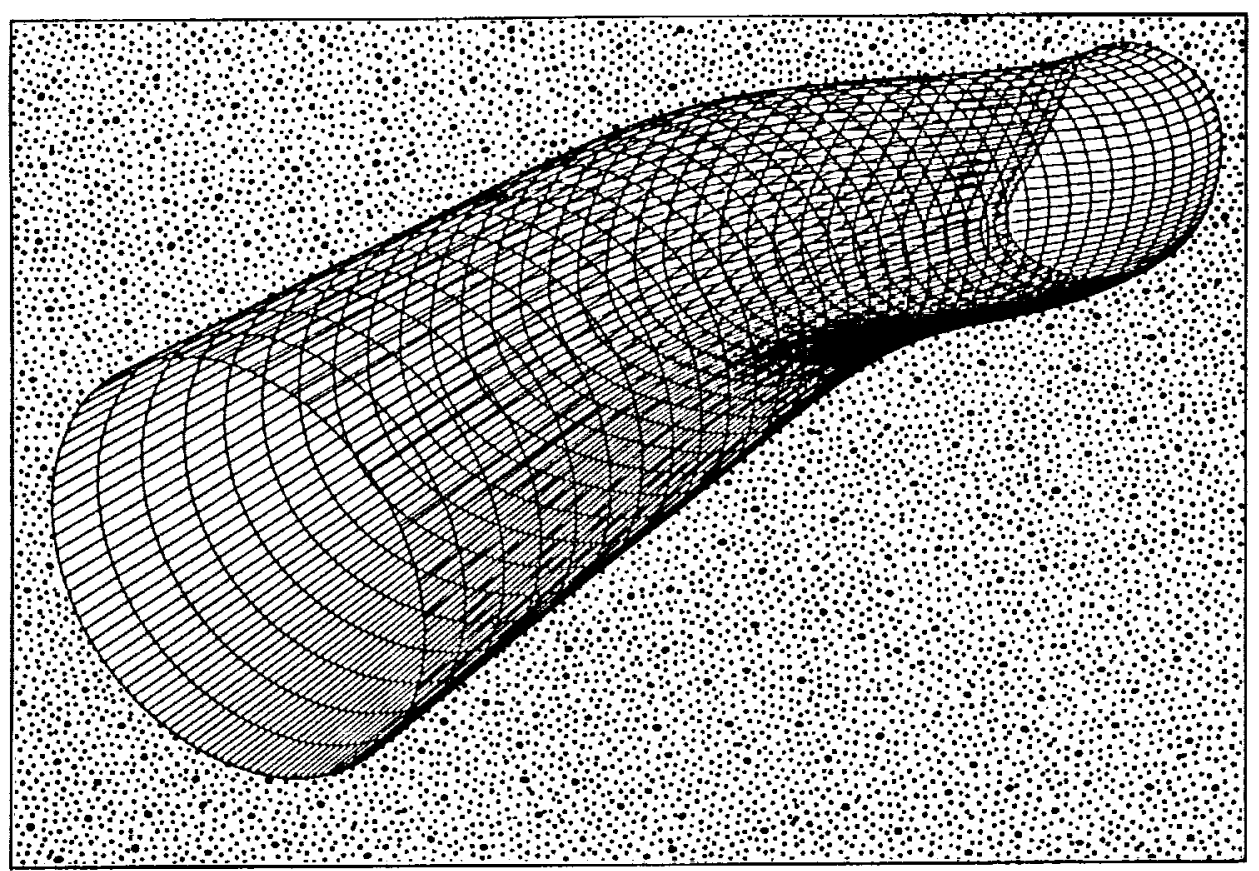

Figure (16) - Computational grid for F/A-18 inlet duct. 


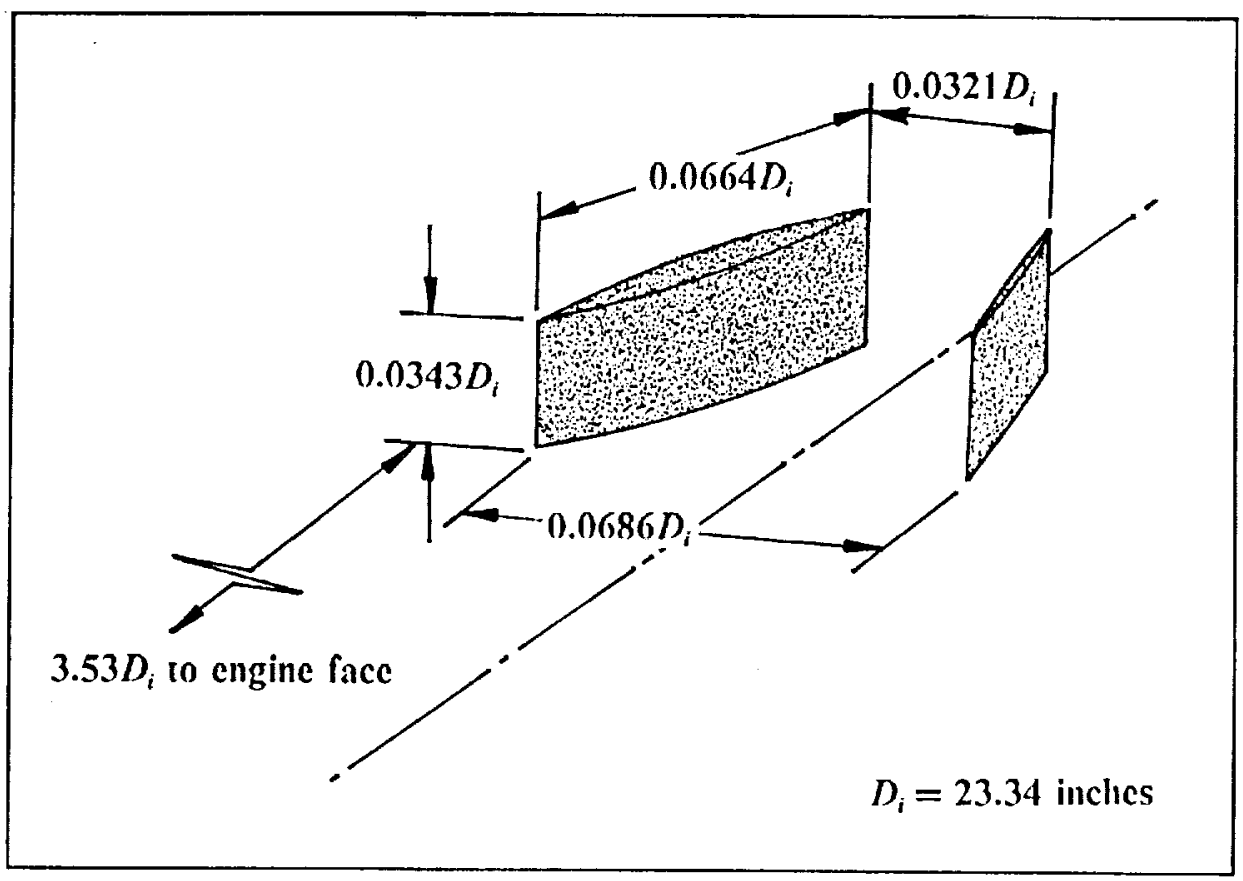

Figure (17) - Location and geometry of vortex generators within F/A-18 inlet duct.

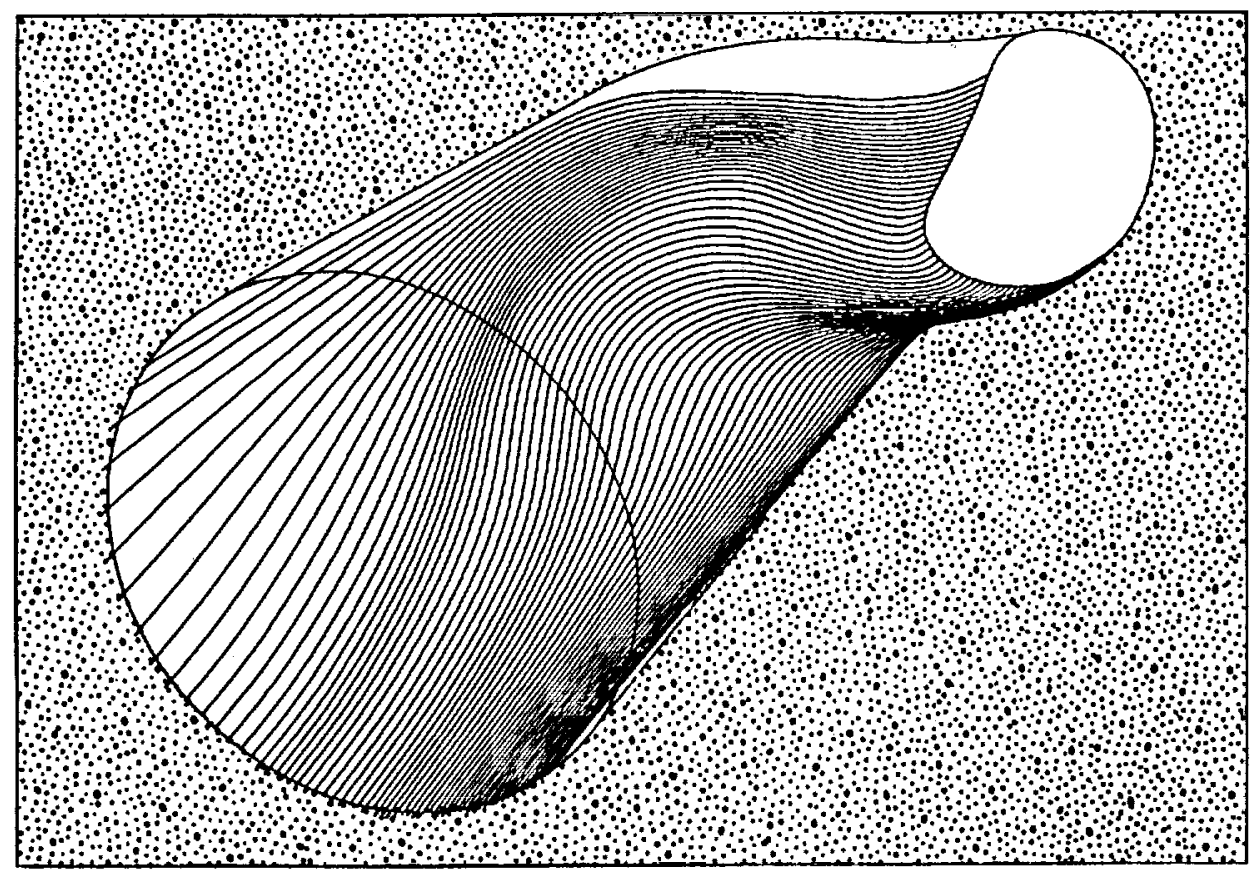

Figure (18) - RNS solution within F/A-18 inlet duct showing limiting streamlines signature without vortex generators. 


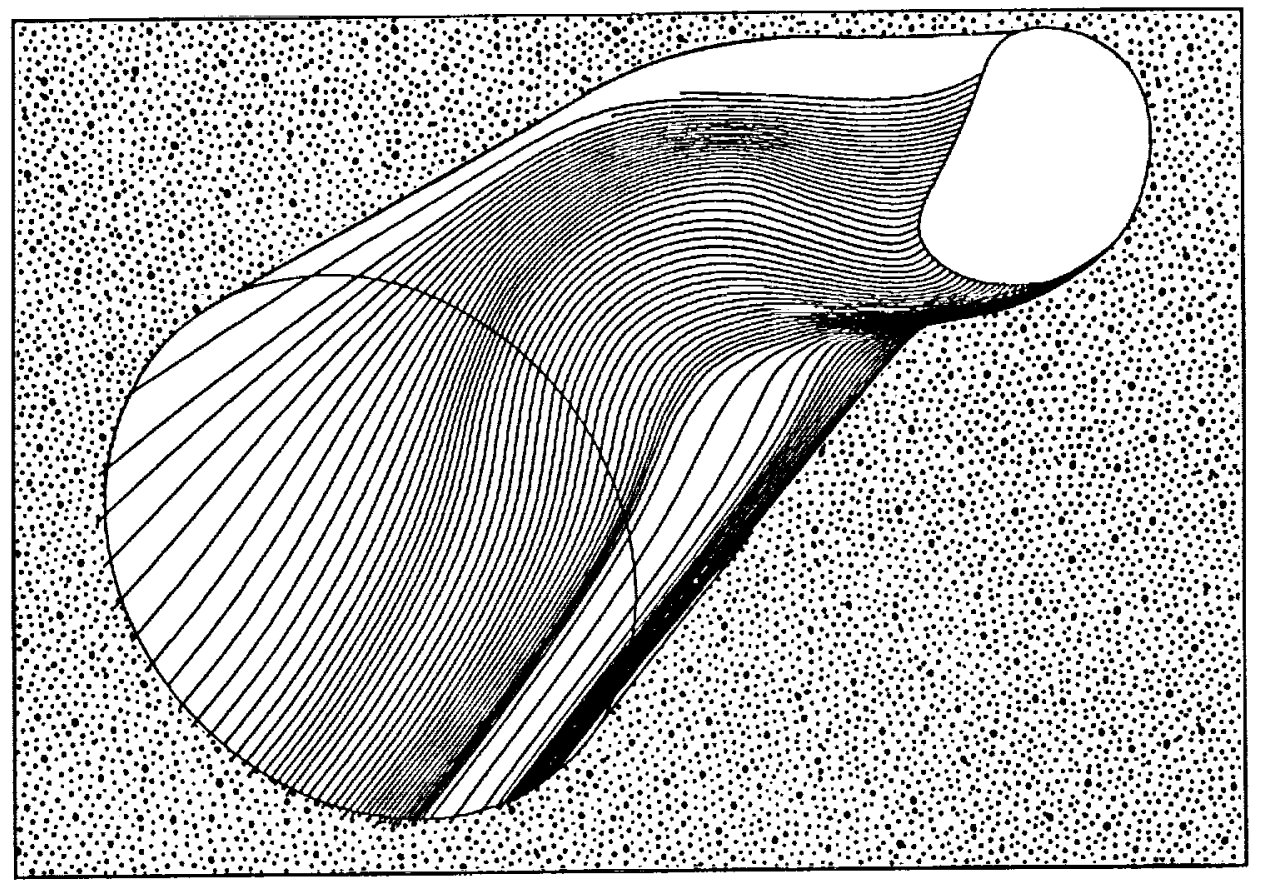

Figure (19) - RNS solution within F/A-18 inlet duct showing limiting streamlines signature with vortex generators.

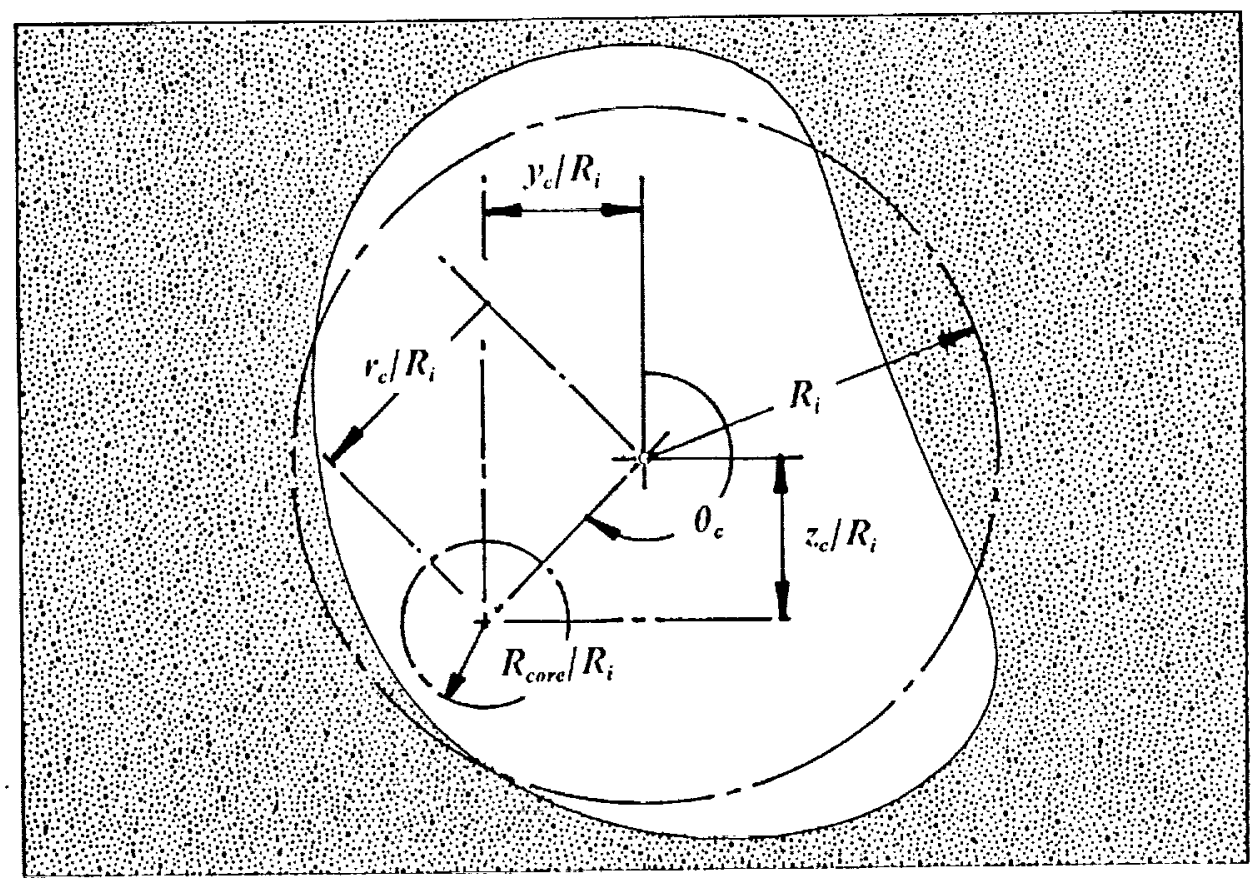

Figure (20) - Nomenclature used for construction of ingested vortex. 


$\begin{array}{cccc}\begin{array}{c}\text { Swirl Angle } \\ \left(\alpha_{s}\right)\end{array} & \begin{array}{c}\text { Core Location } \\ \left(y_{\mathrm{c}} / R_{i}, z_{\mathrm{c}} / R_{i}\right)\end{array} & \begin{array}{c}\text { Core Location } \\ \left(r_{\mathrm{c}} / R_{i}, 0_{\mathrm{c}}\right)\end{array} & \begin{array}{c}\text { Core Radius } \\ \left(R_{\text {carc }} / R_{i}\right)\end{array} \\ 0.0 & -0.551,-0.551 & 0.779,225.0 & 0.291 \\ -5.0 & -0.551,-0.551 & 0.779,225.0 & 0.291 \\ -10.0 & -0.551,-0.551 & 0.779,225.0 & 0.291 \\ -15.0 & -0.551,-0.551 & 0.779,225.0 & 0.291 \\ -20.0 & -0.551,-0.551 & 0.779,225.0 & 0.291 \\ -25.0 & -0.551,-0.551 & 0.779,225.0 & 0.291 \\ -30.0 & -0.551,-0.551 & 0.779,225.0 & 0.291 \\ -35.0 & -0.551,-0.551 & 0.779,225.0 & 0.291 \\ -40.0 & -0.551,-0.551 & 0.779,225.0 & 0.291 \\ -45.0 & -0.551,-0.551 & 0.779,225.0 & 0.291\end{array}$

Table I - F/A-18 inlet duct vortex ingestion test cases.

\begin{tabular}{|cccc|}
\hline & & & \\
$\begin{array}{c}\text { Swirl Angle } \\
\left(\alpha_{s}\right)\end{array}$ & $\begin{array}{c}\text { Core Location } \\
\left(y_{c} / R_{i}, z_{c} / R_{i}\right)\end{array}$ & $\begin{array}{c}\text { Core Locntion } \\
\left(r_{c} / R_{i}, 0_{c}\right)\end{array}$ & $\begin{array}{c}\text { Core Radius } \\
\left(R_{c u r c} / R_{i}\right)\end{array}$ \\
0.0 & $-0.551,-0.551$ & $0.779,225.0$ & 0.291 \\
10.0 & $-0.551,-0.551$ & $0.779,225.0$ & 0.291 \\
20.0 & $-0.551,-0.551$ & $0.779,225.0$ & 0.291 \\
25.0 & $-0.551,-0.551$ & $0.779,225.0$ & 0.291 \\
30.0 & $-0.551,-0.551$ & $0.779,225.0$ & 0.291 \\
40.0 & $-0.551,-0.551$ & $0.779,225.0$ & 0.291 \\
& & & \\
-30.0 & $-0.465,-0.465$ & $0.657,225.0$ & 0.291 \\
-30.0 & $-0.523,-0.523$ & $0.735,225.0$ & 0.291 \\
-30.0 & $-0.551,-0.551$ & $0.779,225.0$ & 0.291 \\
-30.0 & $-0.581,-0.581$ & $0.821,225.0$ & 0.291 \\
-30.0 & $-0.640,-0.640$ & $0.905,225.0$ & 0.291 \\
-30.0 & $-0.698,-0.698$ & $0.987,225.0$ & 0.291 \\
& & & \\
\hline
\end{tabular}

Table II - F/A-18 inlet duct vortex ingestion test cases. 


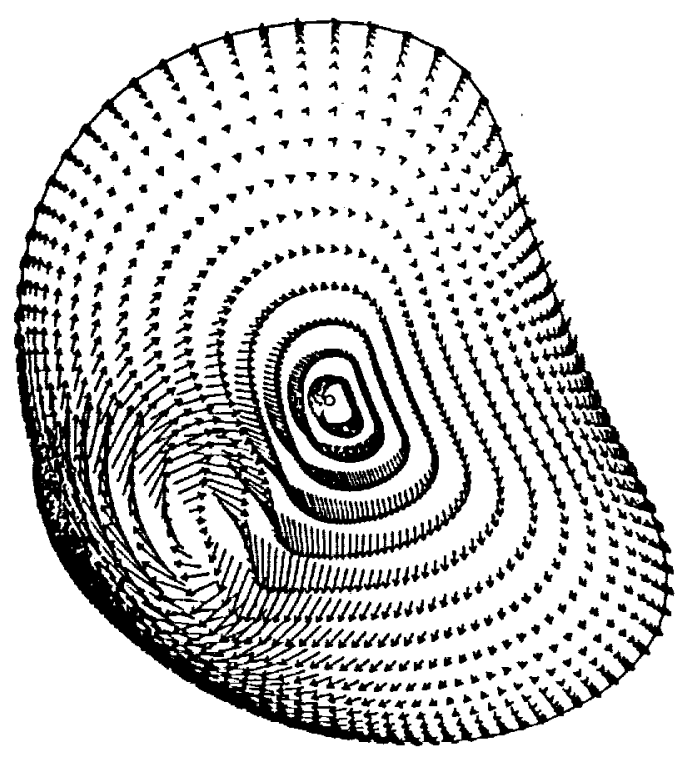

C-VORTEX, $\alpha_{s}=+10.0^{\circ}$

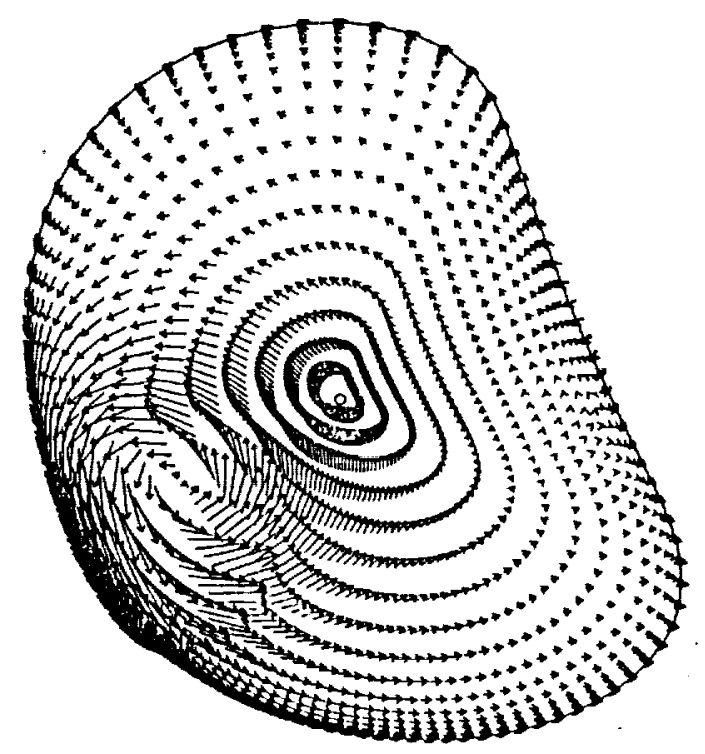

CC-VORTEX, $\alpha_{s}=-10.0^{\circ}$

Figure (21) - RNS solution showing vortex structure within F/A-18 inlet duct, $\mathrm{X} / D_{i}=\mathbf{0 . 0}$.

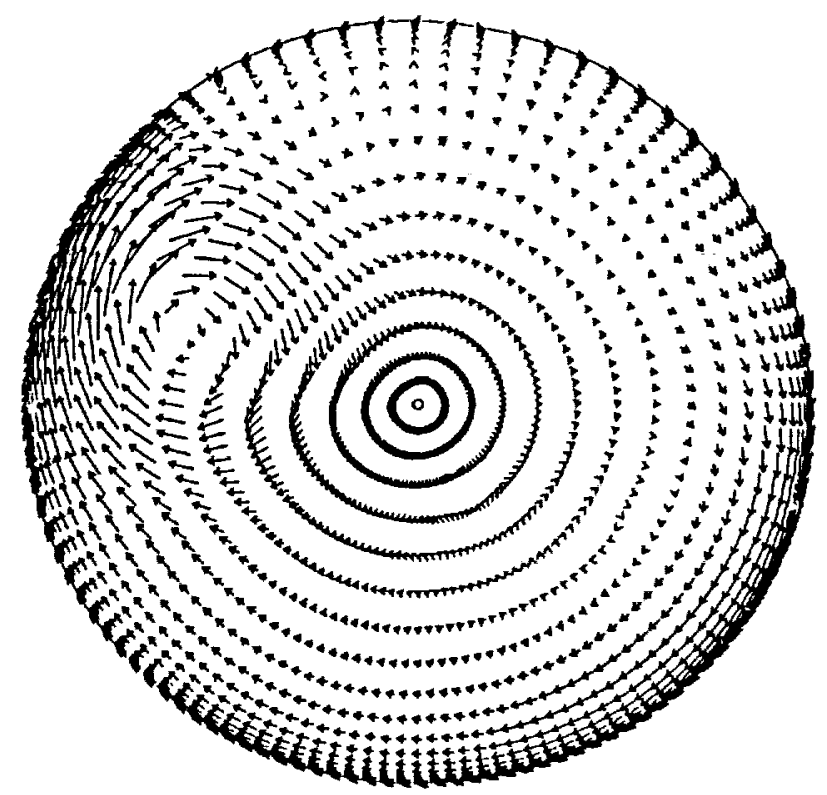

C-VORTEX, $\alpha_{3}=+10.0^{\circ}$

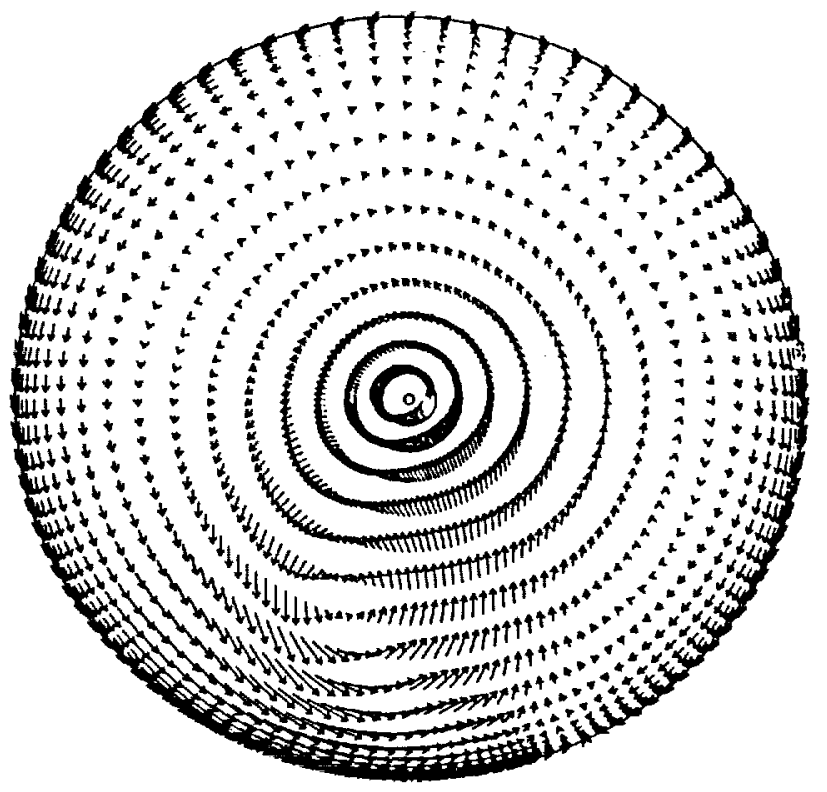

CC-VORTEX, $\alpha_{s}=-10.0^{\circ}$

Figure (22) - RNS solution showing vortex structure within F/A-I8 inlet duct, $\mathrm{X} / D_{i}=6.5$. 


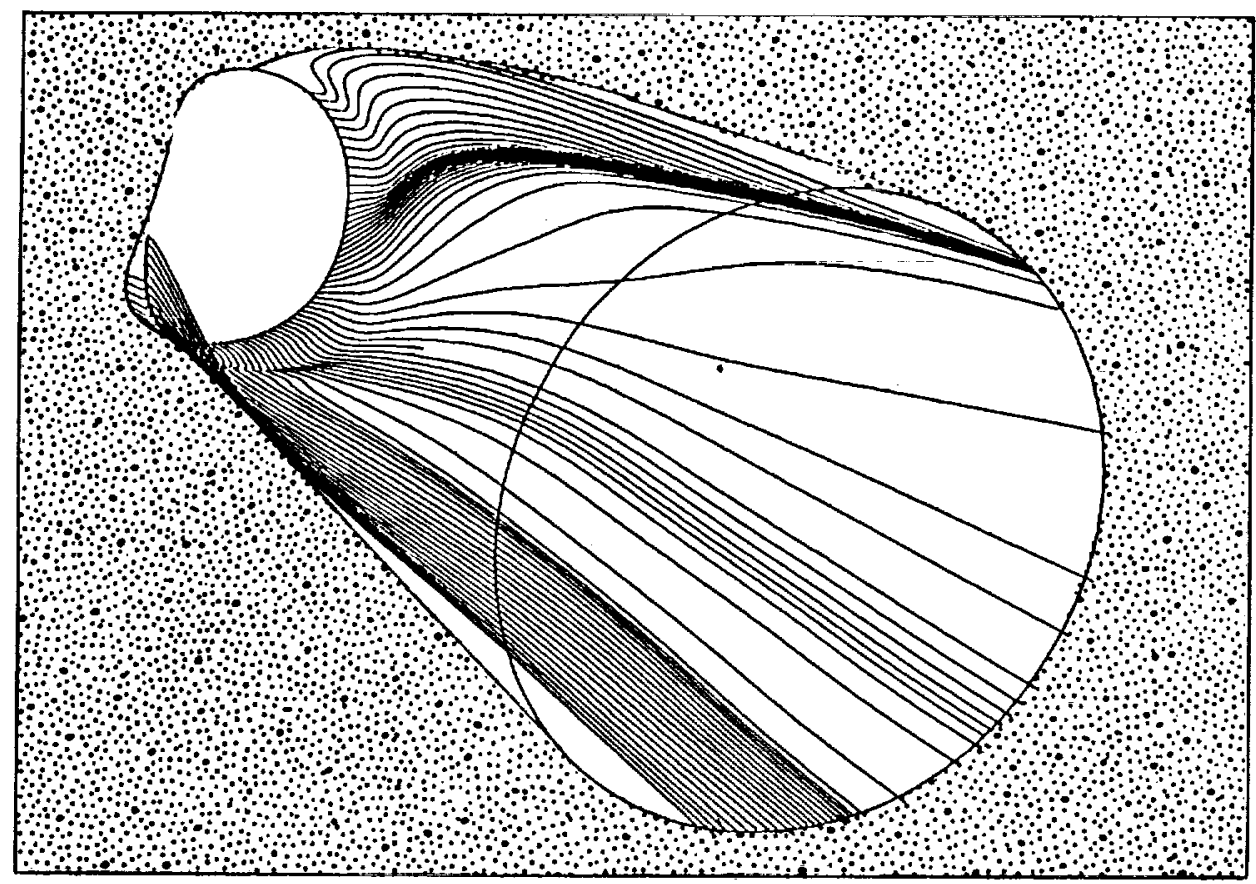

Figure (23) - RNS solution within F/A-18 inlet duct showing limiting streamlines with ingestion of clockwise $(C)$ vortex, $\alpha_{s}=+10.0^{\circ}$.

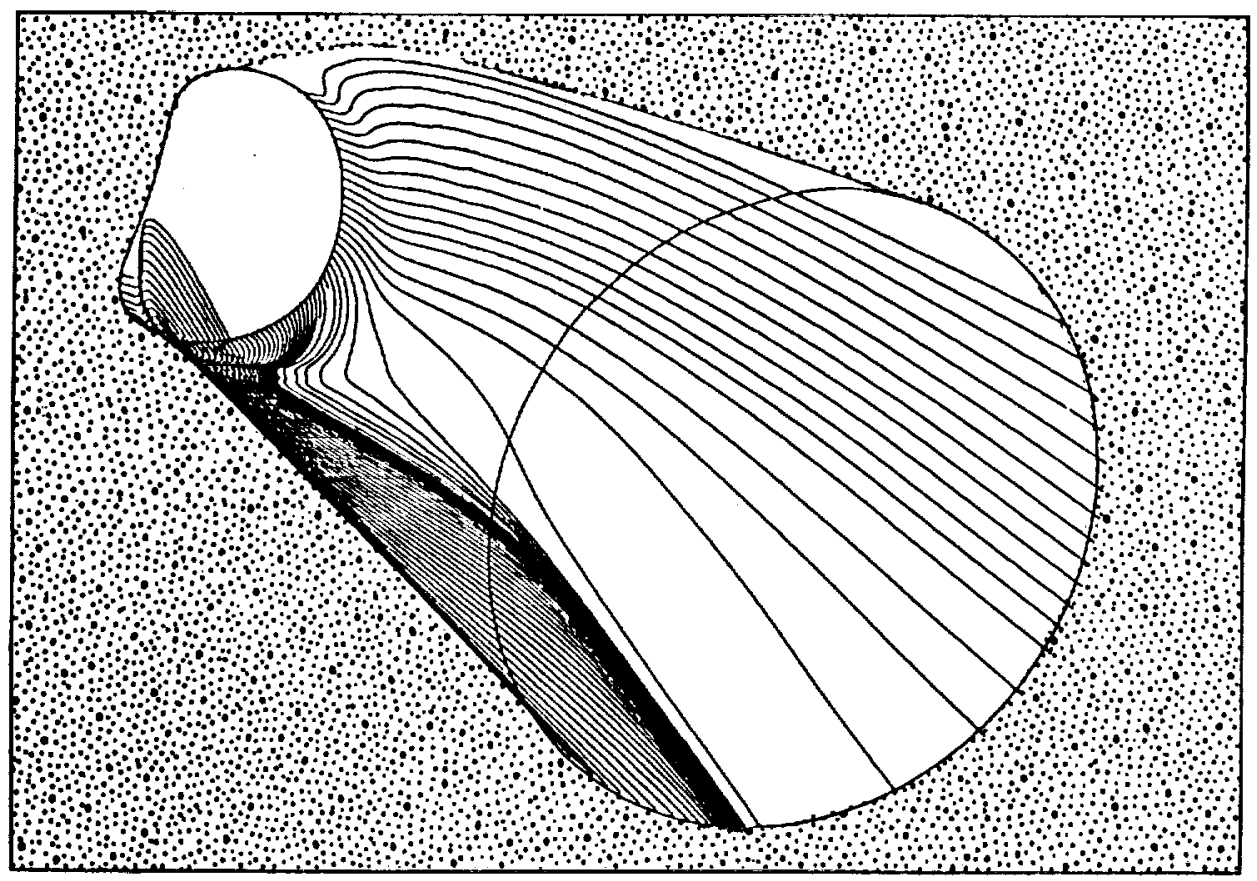

Figure (24) - RNS solution within F/A-18 inlet duct showing limiting streamlines with ingestion of counter clockwise $(\mathrm{CC})$ vortex, $\alpha_{s}=-10.0^{\circ}$. 


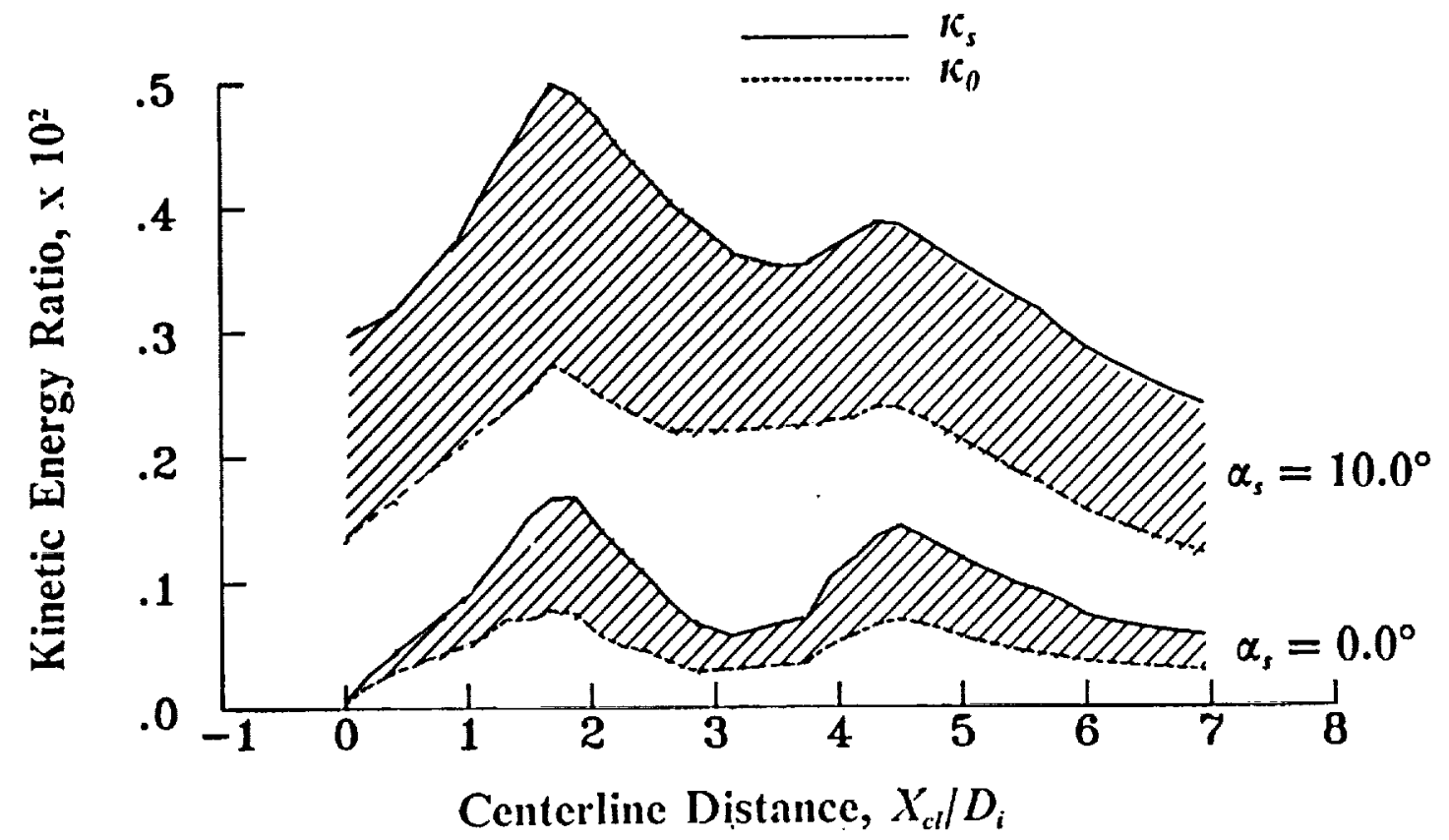

Figure (25) - Distribution of secondary, $\kappa_{s}$, and swirl, $\kappa_{\theta}$, kinetic energy ratio for ingestion of clockwise vortex, $\alpha_{s}=+10.0^{\circ}$.

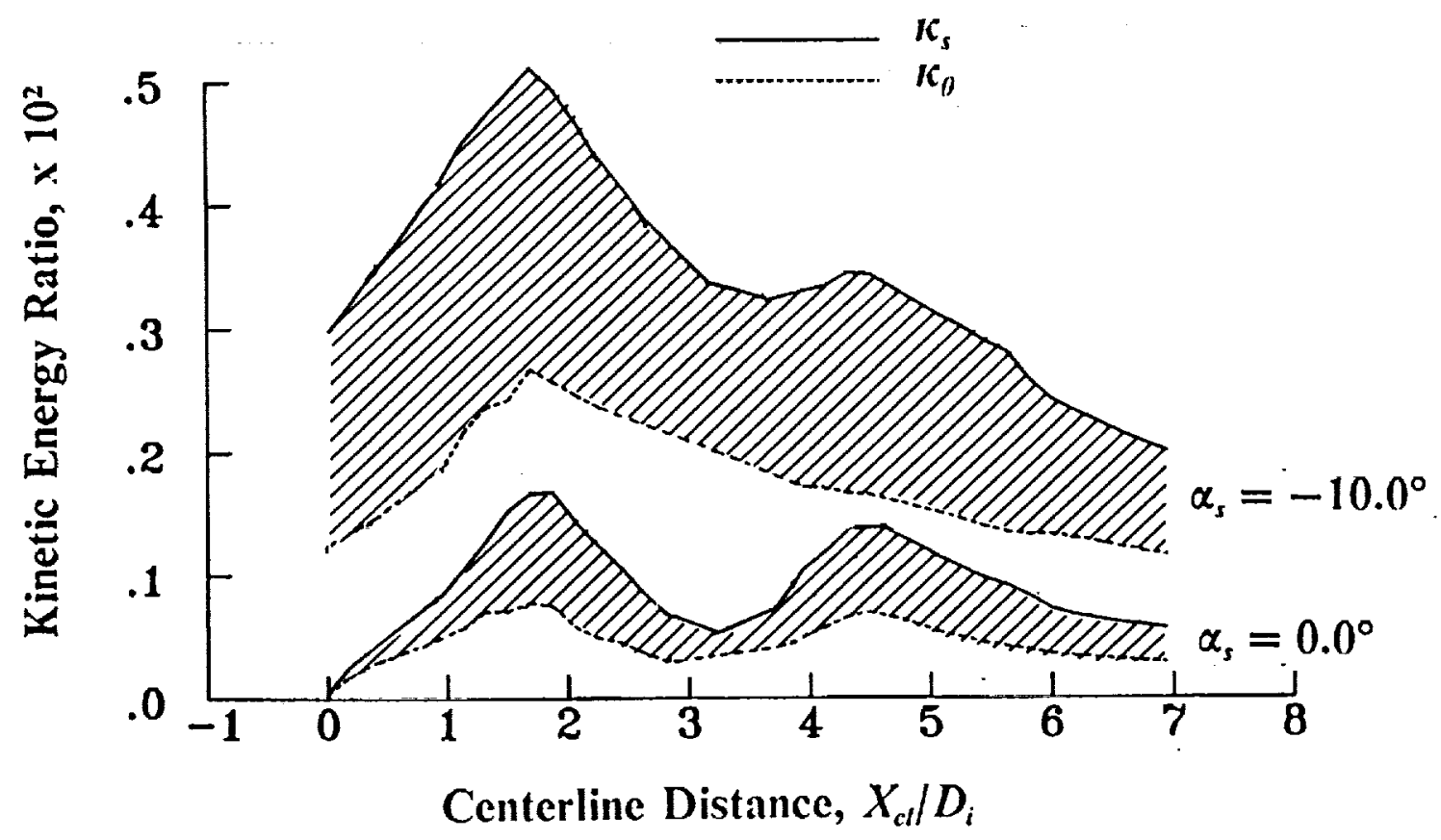

Figure (26) - Distribution of secondary, $\kappa_{s}$, and swirl, $\kappa_{\theta}$, kinetic energy ratio for ingestion of counterclockwise vortex, $\alpha_{s}=-10.0^{\circ}$. 


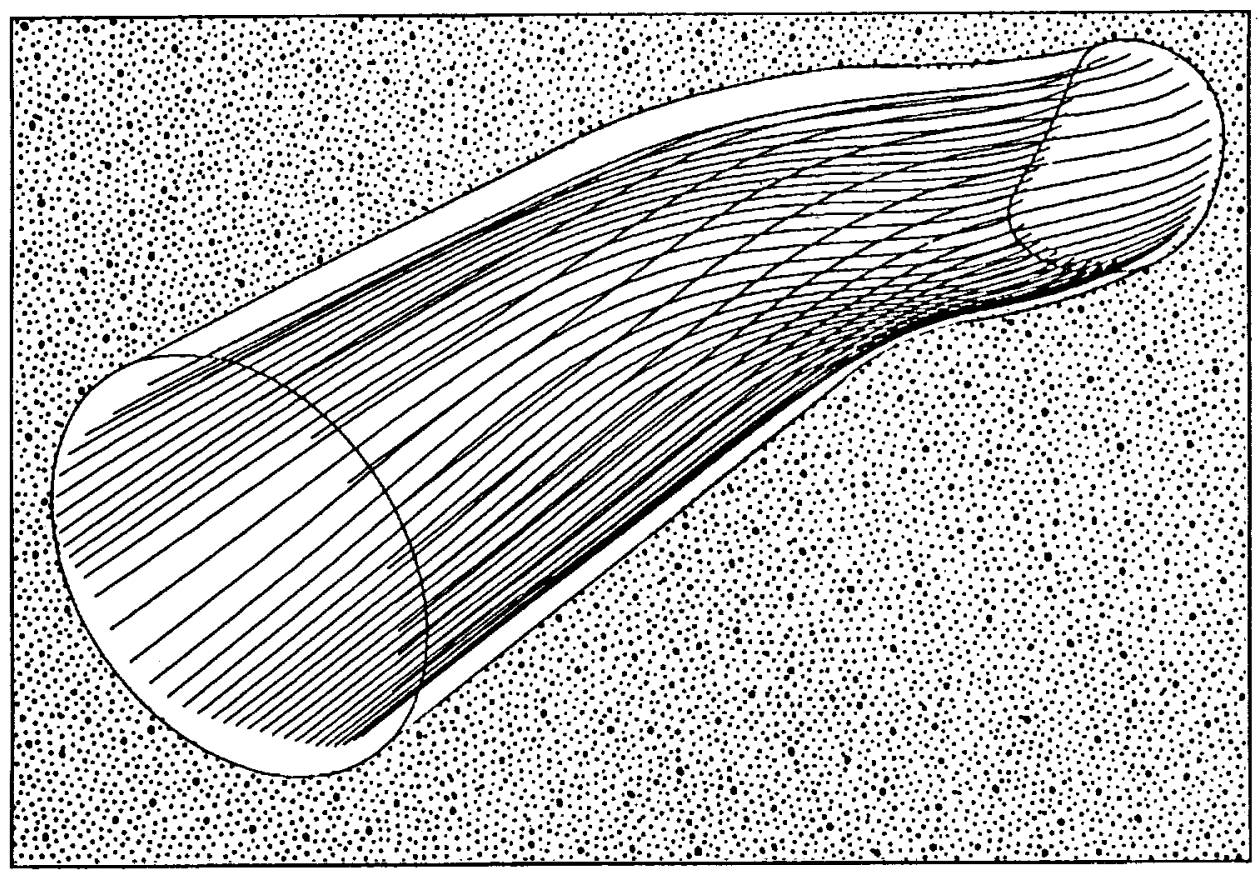

Figure (27) - RNS solution within F/A-18 inlet duct showing particle traces of baseline flow, $y / \delta_{i}=1.14, \alpha_{s}=0.0^{\circ}$.

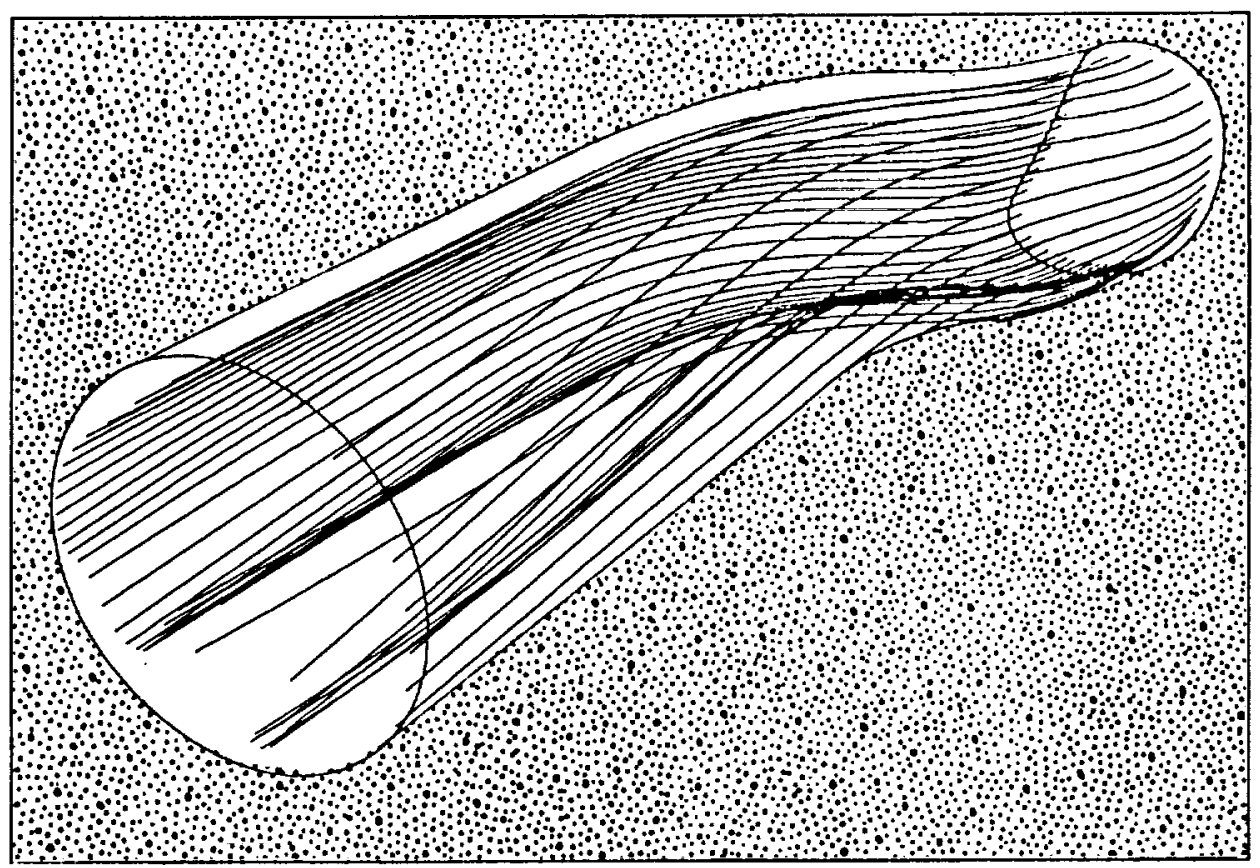

Figure (28) - RNS solution within F/A-18 inlet duct showing particle traces with ingestion of counter clockwise vortex, $\mathrm{y} / \delta_{i}=1.14, \alpha_{s}=-10.0^{\circ}$. 


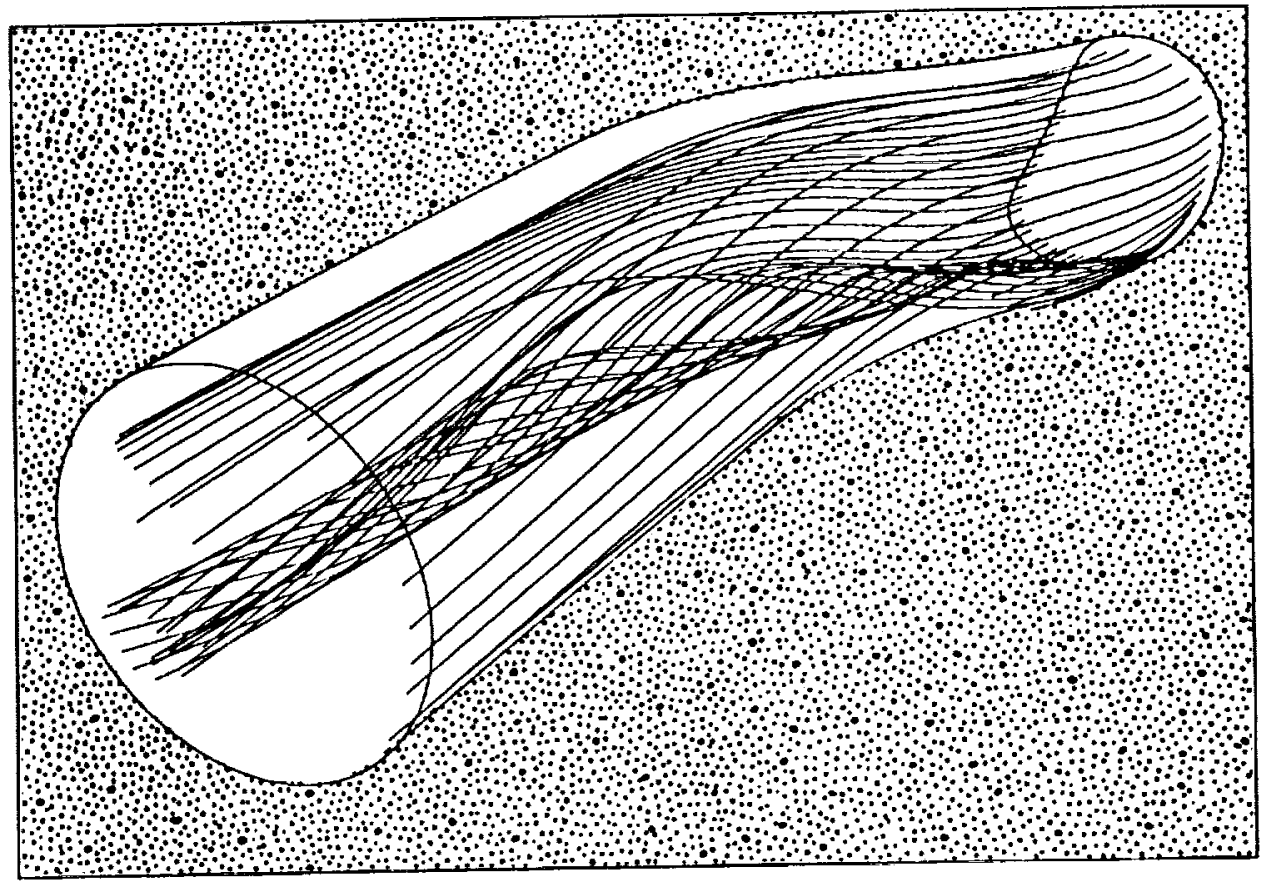

Figure (29) - RNS solution within F/A-18 inlet duct showing particle traces with ingestion of counter clockwise vortex, $\mathrm{y} / \delta_{i}=1.14, \alpha_{s}=-20.0^{\circ}$.

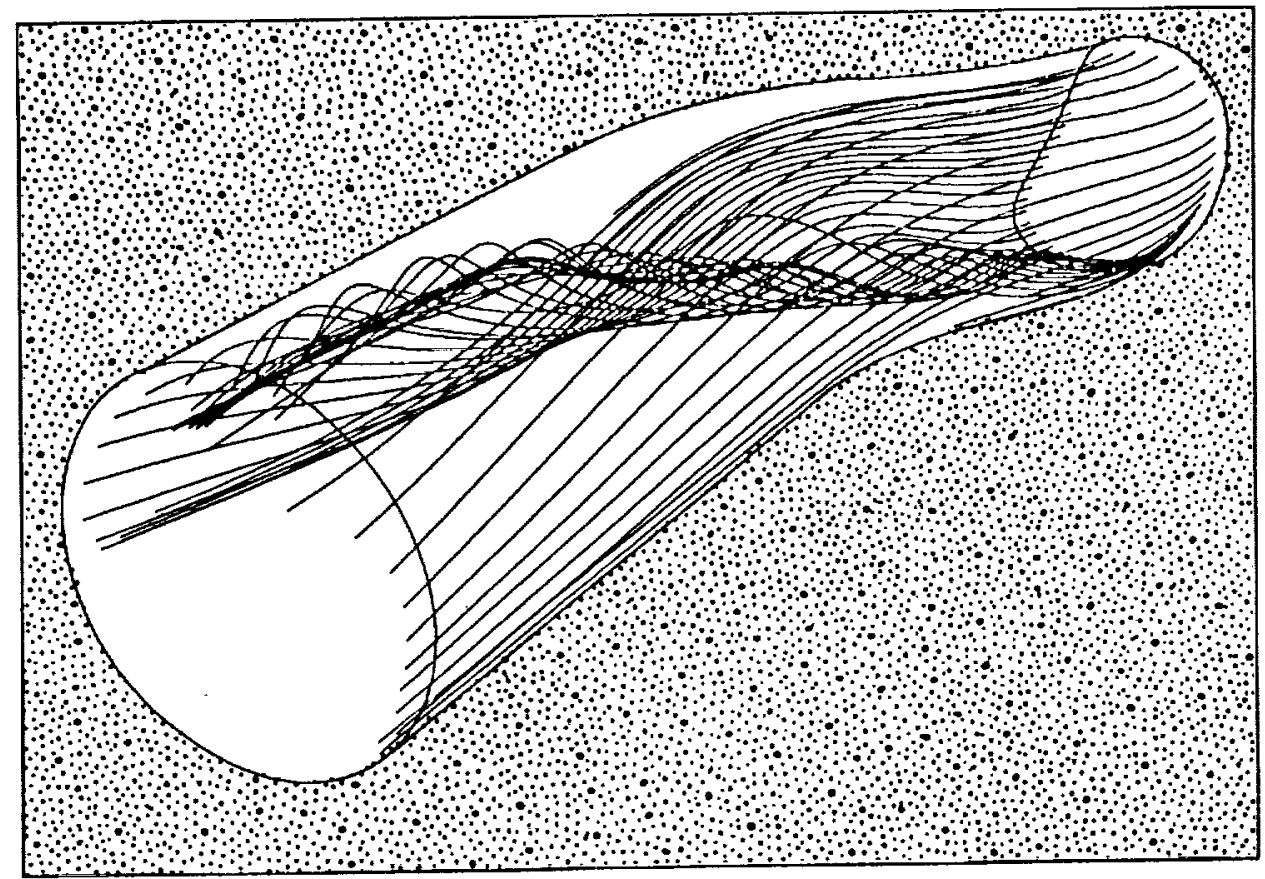

Figure (30) - RNS solution within F/A-18 inlet duct showing particle traces with ingestion of counter clockwise vortex, $y / \delta_{i}=1.14, \alpha_{s}=-30.0^{\circ}$. 


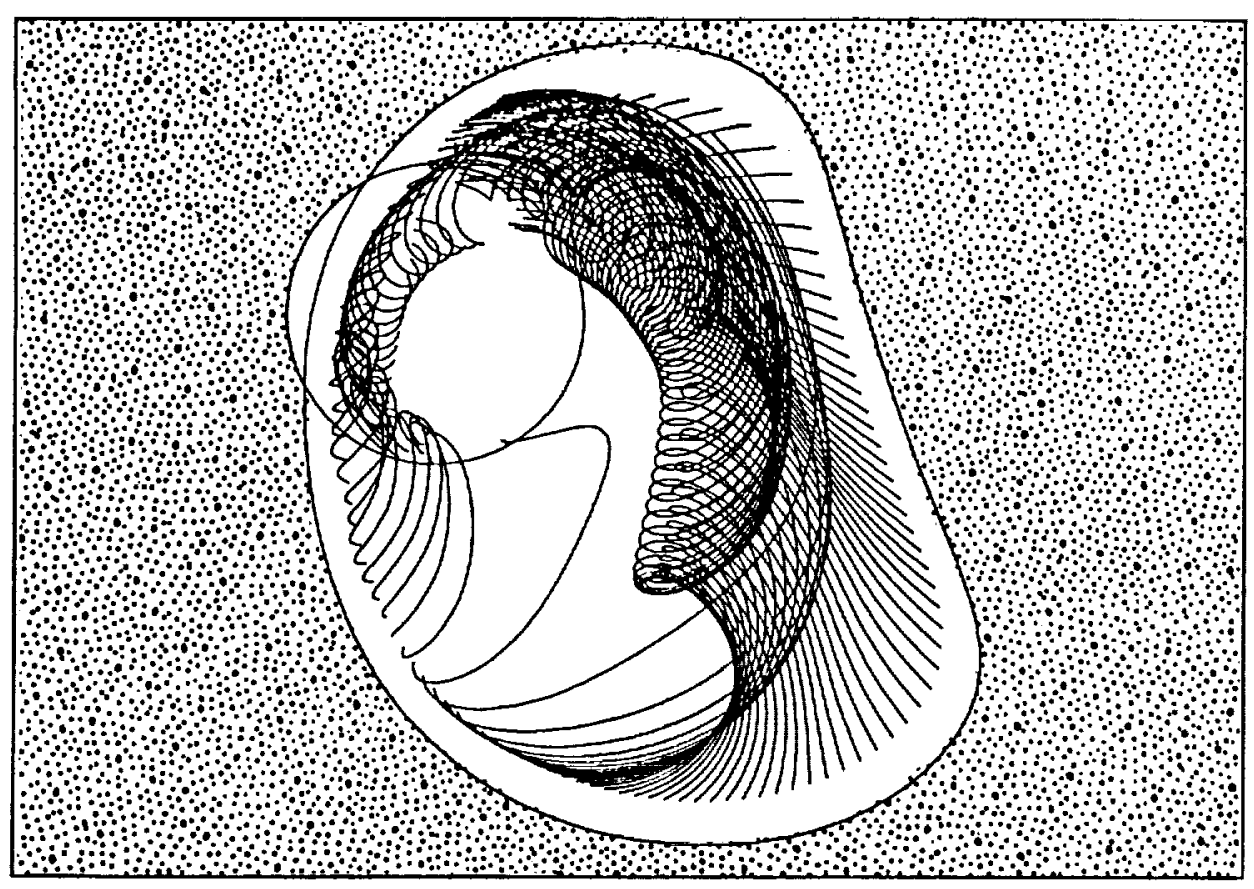

Figure (31) - Downstream view of F/A-18 inlet duct showing particle traces resulting from ingestion of counter clockwise vortex, $y / \delta_{i}=1.14, \alpha_{s}=-40.0^{\circ}$.

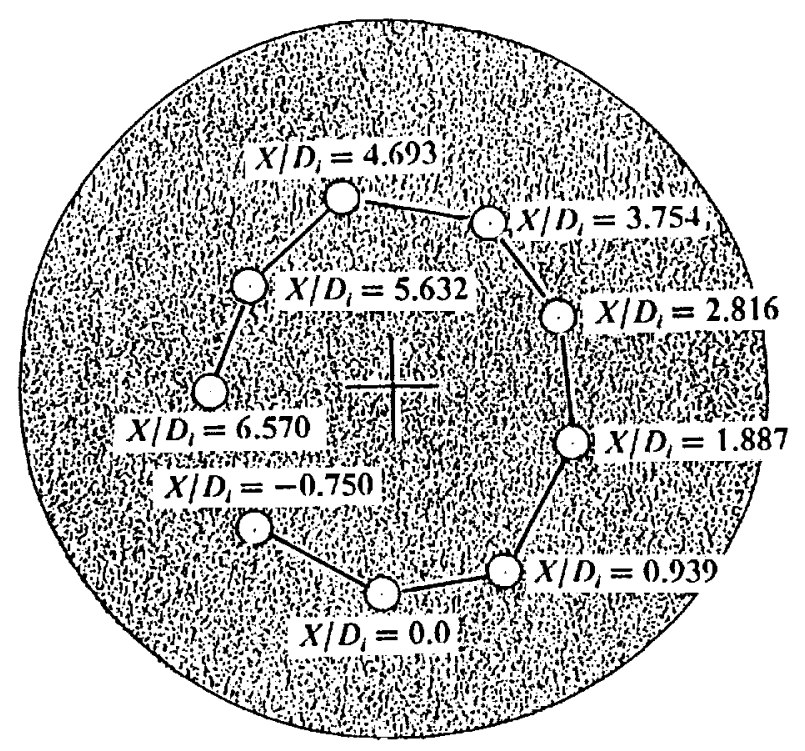

Figure (32) - Computed trajectory of $x_{s}=-40.0^{\circ}$ counter clockwise vortex relative to inlet duct centerline. 


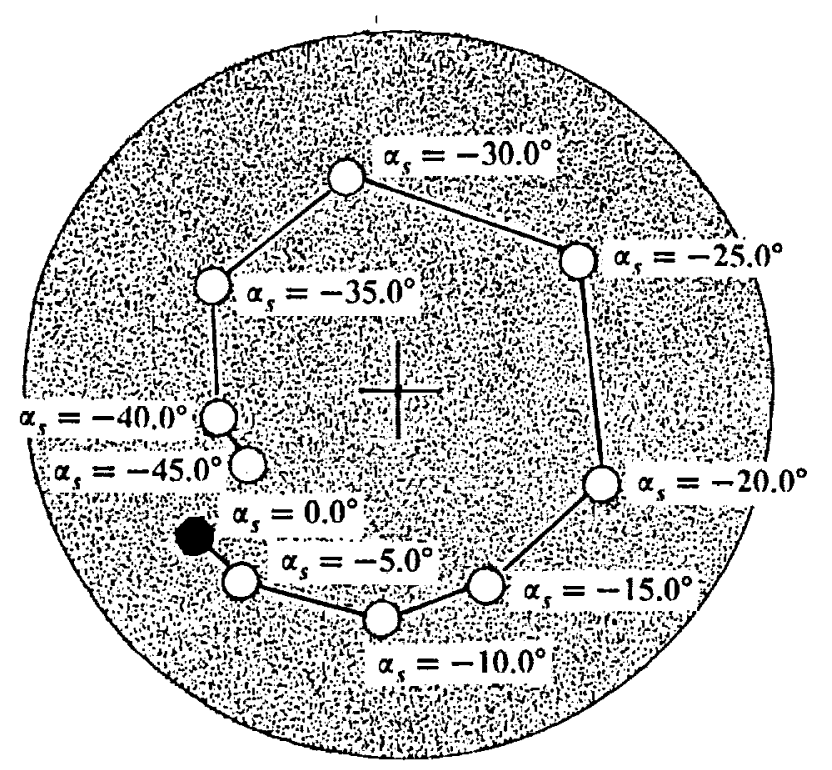

Figure (33) - Effect of vortex strength on impingement location at engine face for ingestion of counter clockwise vortex, $r / R_{i}=0.551$.

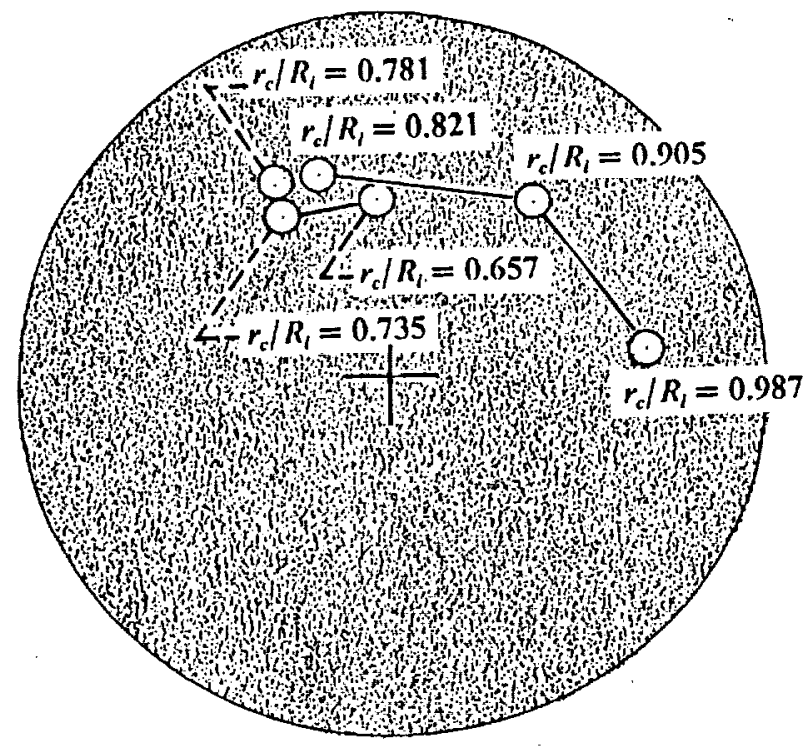

Figure (34) - Effect of vortex entry point on impingement location at engine face for ingestion of counter clockwise vortex, $\alpha_{3}=-30.0^{\circ}$. 


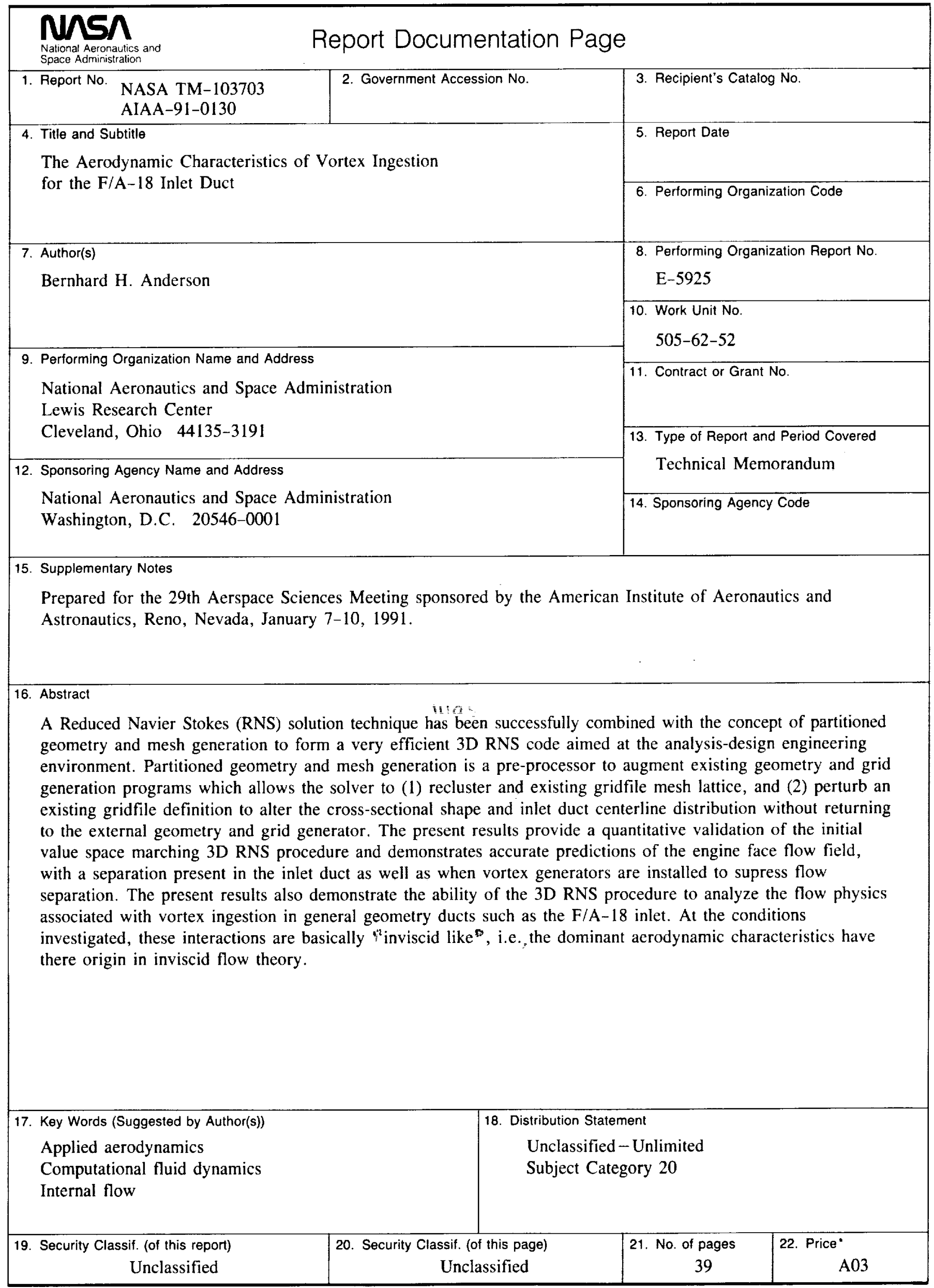


